ABHANDLUNGEN

Berufe und Geschlechterungleichheit

\title{
Der Einfluss der beruflichen Geschlechtersegregation und beruflicher Arbeitszeitarrangements auf Teilzeitarbeit
}

\section{Gleiche Übergangsbedingungen für Frauen und Männer?}

\author{
Agnieszka Althaber · Kathrin Leuze
}

Online publiziert: 19. Mai 2020

(C) Der/die Autor(en) 2020

Zusammenfassung Teilzeitarbeit gilt in Deutschland als ein wichtiges Instrument für die Vereinbarkeit von Beruf und Familie. Bisherige Erklärungen von Teilzeitarbeit fokussieren vorrangig auf Frauen und diskutieren individuelle, haushaltsbezogene und institutionelle Faktoren. Männer sowie berufsstrukturelle Einflussfaktoren auf Teilzeitarbeit wurden bislang jedoch kaum adressiert. In diesem Beitrag untersuchen wir daher die Bedeutung von Berufsmerkmalen für Übergänge von Vollzeit- in Teilzeitbeschäftigung von Frauen und Männern zwischen 1992 und 2015 in Deutschland. Im Fokus steht die Frage, inwiefern die berufliche Geschlechtersegregation und berufliche Arbeitszeitarrangements als Rahmenbedingungen den Übergang in Teilzeit erklären können und ob sie geschlechterdifferente Effekte aufweisen. Unsere theoretischen Überlegungen basieren auf Krügers Institutionenansatz und Ackers Ansatz der Gendered Organizations. Für die Analysen werden Daten des Nationalen Bildungspanels (Startkohorte 6) mit aggregierten beruflichen Merkmalen, basierend auf dem Mikrozensus, kombiniert. Die Ergebnisse der Cox Proportional HazardModelle zeigen, dass weniger die berufliche Geschlechtersegregation, sondern vor allem berufliche Arbeitszeitarrangements Übergänge in Teilzeit beeinflussen, allerdings auf geschlechterdifferenzierte Weise. Während die Vielarbeitsnorm in Berufen, gemessen an Vollzeitarbeit und Überstunden, Teilzeitarbeit für Männer verhindert, gehen Frauen unter diesen Rahmenbedingungen häufiger in Teilzeit über.

\footnotetext{
A. Althaber $(\bowtie)$

Wissenschaftszentrum Berlin für Sozialforschung

Reichpietschufer 50, 10785 Berlin, Deutschland

E-Mail: agnieszka.althaber@wzb.eu

K. Leuze

Friedrich-Schiller-Universität Jena

Carl-Zeiß-Straße 3, 07743 Jena, Deutschland

E-Mail: kathrin.leuze@uni-jena.de
} 
Schlüsselwörter Teilzeitarbeit · Berufe - Arbeitszeitnormen · Geschlechterrollen • Geschlechterungleichheiten · Ereignisdatenanalyse

\title{
The Influence of Occupational Sex Segregation and Occupational Working Time Arrangements on Part-time Work
}

Equal Opportunities for Women and Men?

\begin{abstract}
In Germany, part-time work is considered to be important for reconciling paid work and family responsibilities. Previous explanations of part-time work focused primarily on women and discussed individual, household, and institutional factors. However, men as well as occupational factors influencing part-time work have hardly been addressed so far. In this article, we therefore examine the importance of occupational characteristics for the transitions from full-time to part-time employment for women and men between 1992 and 2015 in Germany. Our main question is: how do occupational gender segregation and working-time arrangements explain the transition to part-time work and do they have gender-differentiated effects? Our theoretical considerations are based on Krüger's institutional approach and Acker's gendered organizations approach. For the analyses data from the National Education Panel Study (Starting Cohort 6) are merged with aggregated occupational characteristics based on the German Microcensus. The results of the Cox proportional hazard models show that rather than occupational sex segregation, it is occupational working-time arrangements that affect transitions to part-time work, but in a gender-differentiated way. While the prevalence of ideal worker norms in occupations, measured in terms of full-time work and overtime, prevent men from working part-time, women change to part-time under these conditions more often.
\end{abstract}

Keywords Part-time work · Occupations · Ideal worker norm · Gender roles · Gender equality $\cdot$ Event history analysis

\section{Einleitung}

Während über die letzten Jahrzehnte eine Angleichung der Bildungs- und Erwerbsbeteiligung von Frauen und Männern in Deutschland stattgefunden hat, haben sich die Geschlechterunterschiede mit Blick auf Teilzeitarbeit vergrößert (Trappe et al. 2015). Teilzeitarbeit ist nach wie vor ein Beschäftigungsmerkmal von Frauen, obwohl auch Männer zunehmend häufiger verkürzte Arbeitszeiten aufweisen (Wanger 2015). So arbeiteten im Jahr $200027 \%$ der sozialversicherungspflichtig beschäftigten Frauen, aber nur 3,5\% der Männer in Teilzeit, bis 2018 stiegen die Teilzeitanteile auf 47,9 bzw. 11,2\% an (Institut Arbeit und Qualifikation 2019). ${ }^{1}$ Der Anteil teil-

\footnotetext{
1 Je nach Datenquelle und Messkonzept werden unterschiedlich hohe Teilzeitquoten berichtet (Wanger 2015). Wir berichten hier Teilzeitquoten von sozialversicherpflichtig Beschäftigten, da diese im Fokus unserer empirischen Analysen stehen. Unter Berücksichtigung von geringfügig Beschäftigten wären die Teilzeitquoten für beide Geschlechter noch deutlich höher (Frauen 57,8\%, Männer 20,2\%, Wanger 2015, S. 2).
} 
zeitbeschäftigter Frauen in Deutschland ist damit einer der höchsten der westlichen industrialisierten Länder, während der Anteil teilzeitbeschäftigter Männer nahe am Durchschnitt liegt (Organisation for Economic Co-operation and Development 2018, S. 33). Als mögliche Erklärungen der Teilzeitlücke zwischen Frauen und Männern hat die bisherige Forschung primär Faktoren auf der Individual- und Haushaltsebene sowie institutionelle Rahmenbedingungen untersucht (Steiber und Haas 2012).

In der Literatur gibt es jedoch auch Hinweise darauf, dass berufliche Merkmale, wie z. B. die berufliche Geschlechterkomposition und damit einhergehende Arbeitszeitarrangements in Berufen, ganz wesentlich darüber bestimmen, ob Arbeitnehmerinnen und Arbeitnehmer in Teilzeit arbeiten oder nicht. Der deutsche Arbeitsmarkt ist, auch im internationalen Vergleich, durch eine hohe berufliche Geschlechtersegregation gekennzeichnet, die sich über die vergangenen Jahrzehnte kaum verringert hat (Hausmann und Kleinert 2014). Mit Blick auf Arbeitszeitarrangements zeigt eine Studie für Deutschland, dass der Anteil regulärer Teilzeitbeschäftigung in Fürsorge- oder Reinigungsberufen $30 \%$ und mehr beträgt, während er in Maschinenund Metallberufen nur 2\% ausmacht (Eichhorst et al. 2013, S. 29). Zudem gehen laut Studien für die USA frauendominierte Berufe häufiger mit Teilzeitarbeit einher (Cassirer 2004), während Mehrarbeit insbesondere in männerdominierten Berufen verbreitet ist (Cha 2013). Allerdings wurden die berufliche Geschlechterkomposition oder Arbeitszeitarrangements bislang selten für die Erklärung von Teilzeitarbeit berücksichtigt, vor allem nicht für Deutschland. Gleichzeitig gibt es erste Hinweise darauf, dass sich diese Berufsmerkmale unterschiedlich auf die Teilzeitbeschäftigung von Frauen und Männern auswirken (Hinz und Schübel 2001), doch auch diese geschlechterdifferenten Effekte wurden bislang kaum systematisch untersucht.

Wir greifen diese zwei Lücken auf und fragen, inwiefern die berufliche Geschlechterkomposition und berufliche Arbeitszeitarrangements den Übergang in Teilzeitbeschäftigung beeinflussen und ob diese Berufsmerkmale unterschiedlich für Frauen und Männer wirken. Berufliche Arbeitszeitarrangements bezeichnen dabei die durchschnittliche Arbeitszeitgestaltung in einem Beruf, z. B. mit Blick auf den Umfang der Arbeitszeit, Überstunden oder die Anwesenheit vor Ort. Deutschland bietet sich für die Untersuchung unserer Fragestellung besonders an, da hier die berufliche Strukturierung des Arbeitsmarkts stark ausgeprägt ist (Solga und Konietzka 1999). Gleichzeitig steht Teilzeitbeschäftigung im Zusammenhang mit verschiedenen Dimensionen von Geschlechterungleichheiten. So ist Teilzeitbeschäftigung einerseits ein wichtiges Instrument für die Vereinbarkeit von Beruf und Familie, vor allem für Frauen (Wanger 2015; Klenner und Schmidt 2011). Andererseits ist Teilzeitbeschäftigung mit einer Reihe von Nachteilen verbunden, insbesondere wenn sie über einen längeren Zeitraum ausgeübt wird (Klenner und Schmidt 2011), wie z.B. mit geringeren Löhnen (Wolf 2014; Bell und Freeman 2001) und geringeren Lohnzuwächsen (Boll 2010), schlechteren Aufstiegsmöglichkeiten (Bell und Freeman 2001), vor allem in Managementpositionen (Hipp und Stuth 2013), was in der Konsequenz das Risiko von Altersarmut erhöht (Frommert und Strauß 2013). Wenn strukturelle Arbeitsmarktbedingungen, wie z.B. Berufsmerkmale, den Übergang von Frauen und Männern in Teilzeitarbeit beeinflussen, womöglich in geschlechterdifferenter Art und Weise, dann sind dadurch auch wei- 
tere Formen von Geschlechterungleichheiten betroffen, die wir in diesem Beitrag aber nicht in den Blick nehmen können.

Im Folgenden geben wir zunächst einen Überblick über den Stand der Forschung zur Teilzeitbeschäftigung von Frauen und Männern. Basierend auf Helga Krügers Überlegungen zur Institutionalisierung von geschlechtertypischen Lebensverläufen (Krüger 1995a, 2001) und Joan Ackers Ausführungen zu Gendered Organizations (Acker 1990) entwickeln wir Hypothesen über den geschlechterdifferenten Einfluss von beruflicher Geschlechterkomposition und beruflichen Arbeitszeitarrangements auf den Übergang in Teilzeitbeschäftigung. Empirisch untersuchen wir mithilfe von Cox Proportional Hazard-Modellen Übergänge von Vollzeit- in Teilzeitbeschäftigung für Frauen und Männer im Zuge von Jobwechseln für den Zeitraum 1992 bis 2015. Diese modellieren wir anhand der Daten des Nationalen Bildungspanels, Startkohorte 6, in Kombination mit aggregierten Indikatoren zu beruflichen Merkmalen basierend auf dem Mikrozensus.

\section{Forschungsstand}

Teilzeitarbeit ist in Deutschland stark reguliert, während sie in anderen Ländern häufig mit prekärer Beschäftigung gleichgesetzt wird (z. B. für die USA Cassirer 2004). So haben Angestellte in Deutschland seit 2001 einen rechtlichen Anspruch darauf, in Teilzeit arbeiten zu können (Teilzeit- und Befristungsgesetz). Zusätzlich besteht seit 1992 im Rahmen von Elternzeitregelungen das Recht, nach familienbedingten Erwerbsunterbrechungen innerhalb von drei Jahren auch mit reduzierter Arbeitszeit auf den früheren Arbeitsplatz zurückzukehren (Bächmann und Gatermann 2017). Teilzeitarbeit umfasst zum einen reguläre Beschäftigungsverhältnisse, d.h. sozialversicherungspflichtige Teilzeitbeschäftigung oder Beamtenverhältnisse in Teilzeit, und zum anderen geringfügige Beschäftigungsverhältnisse (Wanger 2015, S. 2). Da geringfügig Beschäftigte im Monat maximal $450 €$ verdienen können (seit Januar 2013, davor $400 €)$, keine Steuern und Sozialabgaben zahlen und entsprechend nur in sehr geringem Umfang Anspruch auf Sozialleistungen haben, geht diese Beschäftigungsform mit deutlich höheren Erwerbsrisiken einher als reguläre Teilzeitarbeit (Wolf 2014; Klenner und Schmidt 2011).

Neben den eingangs berichteten unterschiedlichen Teilzeitquoten von Frauen und Männern unterscheiden sich auch deren Anteile in regulärer und geringfügiger Teilzeit. Während 2014 die Mehrheit aller teilzeitbeschäftigten Frauen (67\%) reguläre Beschäftigungsverhältnisse innehatte, waren es bei Männern nur knapp die Hälfte (47\%) (Wanger 2015, S. 2). ${ }^{2}$ Darüber hinaus sind Frauen und Männer in unterschiedlichen Lebensphasen teilzeitbeschäftigt. Bei Frauen nimmt der Anteil regulärer Teilzeitarbeit in der familienintensiven Phase bis zum Alter von 49 Jahren kontinuierlich

\footnotetext{
2 Neben Geschlechterunterschieden in der Teilzeitarbeit finden sich auch innerhalb der Gruppe der Frauen große Unterschiede zwischen Ost- und Westdeutschland. So war im Jahr 2014 die Teilzeitquote von Frauen in Westdeutschland mit 48,9\% deutlich höher als in Ostdeutschland (38,4\%), während sie sich für Männer zwischen beiden Teilen Deutschlands nicht unterschied (Rengers 2015). Da der Hauptfokus des Beitrags jedoch auf Geschlechterunterschieden liegt, werden wir Ost-West-Unterschiede nur kontrollieren.
} 
zu und fällt danach langsam wieder ab. Bei Männern ist reguläre Teilzeitarbeit relativ gleichmäßig über die unterschiedlichen Altersgruppen verteilt, mit leicht höheren Anteilen bei den 25- bis 29-Jährigen und bei den 60- bis 64-Jährigen, letzteres vor allem in Form von Altersteilzeit. Geringfügige Teilzeitbeschäftigung kommt dagegen bei beiden Geschlechtern häufig während der Ausbildung (unter 24 Jahren) und nach der Verrentung (über 64 Jahren) vor, bei Frauen zudem ab 45 Jahren, um Pflegetätigkeiten übernehmen zu können (Wanger 2015).

Demzufolge unterscheiden sich auch die Gründe für Teilzeitbeschäftigung stark zwischen den Geschlechtern. Während Frauen am häufigsten aus familiären Gründen in Teilzeit arbeiten, um Kinder oder pflegebedürftige Personen zu betreuen, sind familiäre Verpflichtungen als Teilzeitgründe für Männer eher selten. Stattdessen geben Männer häufiger die Nichtverfügbarkeit einer Vollzeitstelle sowie Ausund Weiterbildung als Grund für ihre reduzierte Erwerbstätigkeit an (Wanger 2015; Klenner und Schmidt 2011). Diese Geschlechterunterschiede machen deutlich, dass wichtige Erklärungsfaktoren für die Teilzeitbeschäftigung auf der Individual- und Haushaltsebene angesiedelt sind (Kühhirt 2012; Wanger 2015; Steiber und Haas 2012; Schober 2013).

Neben der Betreuung von Kindern und pflegebedürftigen Personen sind bei Frauen zudem Prozesse der Selbstselektion in Erwerbstätigkeit generell, aber auch in Teilzeitarbeit, relevant (Steiber und Haas 2012). So bestehen z. B. große Unterschiede zwischen Frauen mit unterschiedlichen Qualifikationsniveaus. Hochqualifizierte Frauen kehren nach der Geburt eines Kindes schneller in den Arbeitsmarkt zurück (Grunow et al. 2011) und reduzieren deutlich seltener ihre Arbeitszeit, selbst wenn sie Mütter oder verheiratet sind, als Frauen mit geringerem Bildungsniveau (Konietzka und Kreyenfeld 2010).

Auf der Haushaltsebene haben innerpartnerschaftliche Ressourcenkonstellationen, z.B. mit Blick auf Bildung, Einkommen oder Berufsstatus, einen Einfluss darauf, ob Frauen und Männer ihre Arbeitszeit reduzieren (Hipp und Leuze 2015), obwohl hier widersprüchliche empirische Befunde vorliegen (Kühhirt 2012; Schober 2013). Dies wird häufig durch kulturelle Geschlechterstereotype erklärt, die auch im Arbeitsmarkt die geschlechtliche Identität von Frauen und Männern reproduzieren (Brines 1994; Kühhirt 2012; Schober 2013). Entsprechend traditioneller Geschlechterrollenvorstellungen wird Männern die Rolle des Haupternährers zugeschrieben, der für den Erwerb des Haushaltseinkommens durch eine Vollzeiterwerbstätigkeit zuständig ist, unabhängig von seinen individuellen Ressourcen. Im Gegensatz dazu wird Frauen die Zuständigkeit für Haushalt und Familie zugeschrieben. Dies geht mit der Erwartung einher, dass Frauen aufgrund der Fürsorgearbeit bei Heirat und der Geburt von Kindern ihre Arbeitszeit reduzieren - wiederum unabhängig von ihren individuellen Ressourcen (Brines 1994). In der Konsequenz führt die Geburt des ersten Kindes selbst in Paaren mit progressiven Geschlechterrollenvorstellungen zu einer Retraditionalisierung der innerpartnerschaftlichen Arbeitsteilung (Bühlmann et al. 2010).

Aus dieser Perspektive ist Teilzeitarbeit insbesondere von Frauen also nicht das Ergebnis eines rationalen Wahlprozesses, sondern von geschlechterstereotypen $\mathrm{Zu}$ schreibungen. Gerade in Westdeutschland wird diese Perspektive durch das nach wie vor weit verbreitete modifizierte männliche Ernährermodell in den Partnerschaften 
reproduziert (Grunow et al. 2006; Trappe et al. 2015). Unterstützt wird dieses Ernährermodell durch eine nicht ausreichende Betreuungsinfrastruktur insbesondere für Kinder unter 3 Jahren und die gemeinsame Besteuerung von Ehepartnerinnen und Ehepartnern (Hipp und Leuze 2015; Grunow et al. 2006). Somit sind auch wohlfahrtsstaatliche Rahmenbedingungen wichtige Erklärungsfaktoren für Teilzeitarbeit, vor allem für die von Frauen.

Dennoch verweisen empirische Belege darauf, dass die tatsächliche Arbeitszeit von Männern und Frauen häufig nicht den individuellen Arbeitszeitwünschen entspricht. Der Arbeitsumfang beider Geschlechter ist in regulären Teilzeittätigkeiten recht ähnlich (im Durchschnitt für Frauen 23,1 Wochenstunden, für Männer 24,2 Wochenstunden) (Wanger 2011). Zudem möchten Frauen und Männer, die Teilzeit arbeiten, eher mehr, und diejenigen, die Vollzeit arbeiten, eher weniger Stunden pro Woche erwerbstätig sein (Wanger 2015; Klenner und Schmidt 2011). Hinzu kommt, dass die Vereinbarkeit von Beruf und Familie inzwischen für Frauen und Männer gleichermaßen wichtig ist (Vandello et al. 2013), weswegen gerade Väter in Deutschland unzufrieden mit ihrer Arbeitszeit sind und gerne mehr Zeit mit den Kindern und weniger Zeit im Job verbringen würden.

Eine mögliche Erklärung für diesen Befund könnte in strukturellen Bedingungen des Arbeitsmarkts liegen. So könnten berufsstrukturelle Merkmale dazu beitragen, dass eine Anpassung der Arbeitszeit an individuelle Wünsche in manchen Berufen schwerer zu realisieren ist als in anderen Berufen. Bislang gibt es jedoch nur wenige Studien zur Bedeutung von Berufsmerkmalen für die Vereinbarkeit von Beruf und Familie. So sind Teilzeitarbeit und flexible Arbeitszeitarrangements in frauendominierten Berufen weiter verbreitet als in männerdominierten Berufen (Althaber 2018; Eichhorst et al. 2013; Cassirer 2004). ${ }^{3}$ Ebenso scheinen Überstunden und Mehrarbeit vor allem in männerdominierten Berufen vorzukommen (Leuze und Strauß 2016; Cha 2013). Studien für die USA zeigen jedoch auch, dass integrierte anstelle von frauendominierten Berufen das höchste Maß an Arbeitszeitflexibilität ermöglichen (Glauber 2011), oder dass kein Zusammenhang besteht (Glass und Fujimoto 1995). Gleichzeitig gibt es erste Hinweise darauf, dass sich diese Berufsmerkmale unterschiedlich auf die Teilzeitbeschäftigung von Frauen und Männern auswirken (Hinz und Schübel 2001).

Insgesamt deuten die Ergebnisse bisheriger Studien darauf hin, dass die berufliche Geschlechterkomposition und damit verbundene Arbeitszeitarrangements in Berufen darüber mitbestimmen, ob Arbeitnehmerinnen und Arbeitnehmer in Teilzeit arbeiten oder nicht. Allerdings wurden diese Berufsmerkmale bislang selten explizit für die Analyse des Übergangs in Teilzeitarbeit berücksichtigt, vor allem nicht für Deutschland. Auch ist nicht ausreichend geklärt, ob diese Berufsmerkmale gleichermaßen für die Teilzeitarbeit von Männern und Frauen relevant sind. Daher werden wir im Folgenden theoretisch diskutieren, wie die berufliche Geschlechterkomposition

\footnotetext{
3 Auch die Geschlechtersegregation in Betrieben spielt diesbezüglich eine Rolle, da in frauendominierten Betrieben die Teilzeitquote deutlich höher ist als in männerdominierten Betrieben (Bechmann et al. 2013, S. 36), und zwar für beide Geschlechter. Aufgrund der Struktur der genutzten Daten können wir jedoch die Betriebsebene nicht identifizieren und fokussieren daher im Folgenden ausschließlich auf Berufe.
} 
und berufliche Arbeitszeitarrangements den Übergang in Teilzeit erschweren oder ermöglichen können.

\section{Theoretische Überlegungen}

Wir nutzen den Institutionenansatz von Helga Krüger (1995a, 2001, 1995b) und den Ansatz der Gendered Organizations von Joan Acker (1990), um zu erklären, wie Berufsmerkmale den Übergang in Teilzeitarbeit beeinflussen können. Beide Ansätze rekurrieren auf die soziale Konstruktion von Geschlecht und darauf, wie kulturelle Vorstellungen traditioneller Geschlechterrollen in Berufsstrukturen eingeschrieben sind und dadurch strukturell unterschiedliche Gelegenheitsstrukturen für Übergänge in Teilzeitbeschäftigung hervorbringen. Diese Gelegenheitsstrukturen können sich zunächst auf den bisherigen Beruf beziehen und Übergänge in Teilzeit ermöglichen oder erschweren. Erst wenn der Wunsch, die Arbeitszeit zu reduzieren, im bisherigen Beruf nicht realisiert werden kann, werden die Gelegenheitsstrukturen in alternativen Berufen eruiert, um gegebenenfalls dorthin zu wechseln. Allerdings sind Berufswechsel im deutschen Arbeitsmarkt aufgrund der engen Kopplung zwischen Ausbildungsberuf und dem ausgeübten Beruf (Solga und Konietzka 1999) eher selten, selbst nach familienbedingten Erwerbsunterbrechungen (Grunow et al. 2011). In den folgenden Ausführungen fokussieren wir daher auf die Gelegenheitsstrukturen des Ausgangsberufs.

Helga Krüger (1995a) nimmt die historische Entwicklung der Geschlechterkomposition von Berufen als Ausgangspunkt ihrer Überlegungen. Sie begreift Berufe als historisch spezifische Organisationen, die nicht nur kulturelle Leitbilder transportieren, sondern auch „Handlungsrahmen, Begrenzungen für Alternativentwürfe und Entscheidungschancen/-zwänge vorgeben“" (Krüger 1995a, S. 196f.). Mit der historischen Entstehung der modernen Berufsstruktur in der ersten Hälfte des 20. Jahrhunderts in Deutschland wurden zunehmend Beschäftigte in personenbezogenen Dienstleistungsberufen, Büro und Verwaltung gebraucht, für die meist junge, unverheiratete Frauen eingestellt wurden (Krüger 1995a, 2001). Da Arbeitgeberinnen und Arbeitgeber davon ausgingen, dass die weiblichen Angestellten nur bis zur Heirat und Familiengründung arbeiten, waren diese Berufe stark vom übrigen Arbeitsmarkt abgegrenzt und boten weder Aufstiegs- noch gute Verdienstmöglichkeiten (Krüger 1995b). Die Ausbildung erfolgte meist durch das in der Bismarck-Ära geschaffene System schulischer Berufsausbildungen, das weitgehend zur Überbrückung des Zeitraums zwischen Schulabschluss und Heirat gedacht war (Krüger 2001). Das so entstandene Berufssegment für (junge) Frauen war daher das Ergebnis institutionalisierter Geschlechterrollen, das eine Vereinbarkeit von Ehe, Familie und Beruf ermöglichen sollte.

Aufgrund von institutionellen Pfadabhängigkeiten besteht diese Ausrichtung von Berufskontexten heute weitgehend fort, obwohl sich die individuellen Einstellungen und Wünsche von Frauen und Männern zu ihrer Lebensführung inzwischen deutlich gewandelt haben (Krüger 2001). So geht die berufliche Geschlechterkomposition auch heute damit einher, dass typisch weibliche und männliche Aufgaben und Arbeitsbereiche über Berufe bestimmt werden, die eng mit den traditionellen 
Geschlechterrollen zusammenhängen (Krüger 1995b). Dies führt dazu, dass die Organisationsprinzipien von Berufen und die Erwartungen an die Verfügbarkeit für den jeweiligen Beruf immer noch weitgehend nach den traditionellen Leitbildern von männlichen Familienernährern und weiblichen Familienfürsorgerinnen ausgerichtet sind (Krüger 1995a). Dementsprechend sind männerdominierte Berufe eher für eine traditionell männliche Geschlechterrolle und für eine Lebensführung konzipiert, die eine kontinuierliche Erwerbstätigkeit in Vollzeit über den gesamten Erwerbsverlauf hinweg vorsehen (Krüger 1995a). Die Organisationsprinzipien und Verfügbarkeitserwartungen in männerdominierten Berufen sollten somit eine deutliche Barriere für Übergänge in Teilzeitarbeit darstellen. Frauendominierte Berufe sind hingegen nach wie vor auf eine traditionell weibliche Geschlechterrolle und eine Lebensführung ausgerichtet, die Diskontinuitäten im Erwerbsverlauf, wie zum Beispiel durch Teilzeitarbeit oder familienbedingte Erwerbsunterbrechungen, berücksichtigen (Krüger 1995a). Folglich ist Teilzeitarbeit in frauendominierten Berufen aufgrund der dort gegebenen Organisationsprinzipien und Verfügbarkeitserwartungen vergleichsweise weit verbreitet. Daher sollte die berufliche Geschlechterkomposition als Gelegenheitsstruktur sowohl für Frauen als auch für Männer den Übergang in Teilzeit beeinflussen.

Hla: Je höher der Frauenanteil im Beruf ist, desto höher sind die Übergangsraten in Teilzeit.

Allerdings bestehen möglicherweise geschlechterdifferente Effekte der beruflichen Geschlechterkomposition. Für Frauen, und insbesondere für Mütter, ist ein Wechsel in Teilzeitarbeit aufgrund ihrer kulturellen Zuständigkeit für Haushalt und Familie gesellschaftlich akzeptiert, zunächst einmal unabhängig von der Geschlechterkomposition in Berufen. Doch durch die unterschiedlichen Gelegenheitsstrukturen in Berufen haben Frauen in frauendominierten Berufen häufiger die Möglichkeit, in Teilzeit zu wechseln. In männerdominierten Berufen gehen Frauen hingegen seltener in Teilzeit über. Dort setzen sie stattdessen andere Vereinbarkeitslösungen um oder wechseln gegebenenfalls in andere Berufe, die eine bessere Vereinbarkeit ermöglichen.

Männern wird dagegen gesellschaftlich nach wie vor die Ernährerrolle zugeschrieben, mit entsprechenden Erwartungen an eine Vollzeiterwerbstätigkeit über den gesamten Lebensverlauf hinweg. Da diese kulturellen Geschlechterstereotypen in männerdominierte Berufe ebenso eingeschrieben sind, sollten Männer in Übereinstimmung mit ihrer Geschlechterrolle aus männerdominierten Berufen seltener in Teilzeit wechseln. Allerdings könnte dies auch in frauendominierten Berufen der Fall sein. Da die Arbeit in frauendominierten Berufen nicht mit der individuellen Geschlechterrolle von Männern übereinstimmt, könnten Männer versucht sein, diesen Widerspruch zwischen institutioneller und individueller Geschlechterrolle auszugleichen, indem sie gerade dort seltener in Teilzeit wechseln. Dies stützen Befunde von Hinz und Schübel (2001), denen zufolge Männer in frauendominierten Berufen häufiger Vollzeitpositionen besetzen als Frauen. In der Konsequenz bedeutet dies, dass Männer in männerdominierten Berufen generell weniger Möglichkeiten haben, in Teilzeitbeschäftigung zu wechseln, während Männer in frauendominierten Berufen 
die vorhandenen Möglichkeiten aufgrund individueller Geschlechterrollenzuschreibungen selten wahrnehmen.

H1b: Ein höherer Frauenanteil im Beruf sollte für Frauen einen stärkeren Einfluss auf den Übergang in Teilzeit haben als für Männer.

Aus der Perspektive des Institutionenansatzes von Helga Krüger bedingt vor allem die historisch gewachsene Geschlechterkomposition des Berufs den Handlungsrahmen und damit die Gelegenheitsstruktur für den Übergang in Teilzeit. Doch ihre empirischen Analysen zeigen auch, dass selbst innerhalb der Gruppe der frauendominierten Berufe Unterschiede in der Nutzung von Teilzeitarbeit bestehen, die sich durch die „Nähe vs. Ferne des erlernten Berufs zu ,männlichen“ Berufskonstrukten“ begründen lassen (Krüger 1995b, S. 147). Dies deutet darauf hin, dass weitere Berufsmerkmale, neben und unabhängig von der beruflichen Geschlechterkomposition, den Übergang in Teilzeit beeinflussen sollten. Allerdings bietet Krügers Institutionenansatz keine theoretischen Anhaltspunkte dafür, wie diese Nähe oder Ferne zu männlichen Berufskonstrukten zu konzeptualisieren ist. Daher nutzen wir ergänzend Joan Ackers Theorie der Gendered Organizations (1990), um weiterführende Annahmen zur Bedeutung von beruflichen Arbeitszeitarrangements für den Übergang in Teilzeit zu generieren.

Ähnlich wie Krügers institutionentheoretische Überlegungen geht der Ansatz der Gendered Organizations (Acker 1990) davon aus, dass sich die Strukturen und Prozesse von Organisationen - einschließlich der Organisation von Arbeitsplätzen am Leitbild der traditionellen männlichen Geschlechterrolle orientieren. In der Konsequenz stellen männlich typisierte Verfügbarkeits- und Verhaltenserwartungen an Arbeitnehmerinnen und Arbeitnehmer die Basis für organisationale Routinen dar und erhalten dadurch einen normativen Charakter. Dieser organisationalen Logik zufolge werden Beschäftigte, die ihr Leben auf bezahlte Erwerbsarbeit ausrichten, die ständig vor Ort sind oder Überstunden leisten, als besser geeignet und als leistungsbereiter wahrgenommen (Acker 1990, S. 149). Somit leiten sich aus diesen Verfügbarkeitserwartungen auch Bewertungen der individuellen Leistung und Leistungsbereitschaft von Beschäftigten ab. Beschäftigte, die den Verfügbarkeitserwartungen entsprechen, erfahren eine höhere Wertschätzung am Arbeitsplatz, haben höhere Löhne und schnellere berufliche Aufstiege als diejenigen, die diese Norm nicht erfüllen (Acker 1990).

In der Literatur werden diese männlich typisierten Verfügbarkeitserwartungen und die mit ihr einhergehenden beruflichen Vor- und Nachteile als Ideal Worker Norm bezeichnet (Williams et al. 2013). Den Kern der Ideal Worker Norm bilden drei Verhaltenselemente, die allgemein als Ausdruck und als Signale von ausgewiesener Leistungsbereitschaft am Arbeitsplatz verstanden werden: (1) lange Arbeitszeiten, (2) die Bereitschaft, auch unvorhergesehene Aufgaben zeitnah zu erledigen, und (3) die physische Anwesenheit am Arbeitsplatz, die mit einer sichtbaren Geschäftigkeit verbunden ist (Kelly et al. 2010, S. 287). Diese drei Verhaltenselemente haben sich wiederkehrend in verschiedenen Organisationskontexten als relevant erwiesen (Williams et al. 2012, 2013). Auch wenn die Ideal Worker Norm in Strukturen und Prozessen der jeweiligen Organisationen verankert ist, variiert sie doch insbesondere 
zwischen Berufen: Für vergleichbare Berufspositionen in unterschiedlichen organisationalen Kontexten fällt die Ideal Worker Norm sehr ähnlich aus (Williams et al. 2012). So sind die Verfügbarkeitserwartungen beispielsweise in männerdominierten Berufen oder in Berufen stärker ausgeprägt, die innerhalb der Organisationshierarchie höher angesiedelt sind (Klenner und Lott 2016). Insofern sind Berufe geeignete Kontexteinheiten, um die Variation der Ideal Worker Norm abzubilden (Leuze und Strauß 2016).

Da Teilzeitbeschäftigung eine klare Abweichung von den Verfügbarkeitserwartungen der Ideal Worker Norm darstellt, gehen damit verschiedene Formen von beruflichen Nachteilen im Vergleich zu Beschäftigten in Vollzeit einher (Wolf 2014; Bünning 2016). Bei hoch qualifizierten Tätigkeiten, in denen die Ideal Worker Norm besonders stark ausgeprägt ist, kann das sogar dazu führen, dass Teilzeitbeschäftigung innerhalb der Organisationen informell nicht als vollwertige Arbeit angesehen wird und ihre Legitimität in Frage gestellt wird (Fuchs Epstein et al. 1999). Doch die beruflichen Nachteile von Teilzeittätigkeiten treten nicht in allen Berufen gleichermaßen auf: In Berufen mit umfassenden Verfügbarkeitserwartungen fallen berufliche Nachteile besonders stark aus, z. B. mit Blick auf Löhne oder Aufstiegschancen (Williams et al. 2013).

Die drei Verhaltenselemente der Ideal Worker Norm lassen sich über die Verbreitung von Vollzeitarbeit, Mehrarbeit sowie der erforderlichen Präsenz am Arbeitsplatz in Berufen abbilden. Es kann angenommen werden, dass Beschäftigte in Berufen seltener in Teilzeit übergehen, in denen Vollzeitarbeit, Mehrarbeit und Präsenz vor Ort die Norm darstellen, um diesen Normen zu entsprechen. Hinzu kommt, dass in diesen Berufen eine Abweichung von der Norm mit höheren Nachteilen einhergeht. Somit ist zu erwarten, dass in Berufen mit einer stärkeren Ideal Worker Norm Übergänge in Teilzeitbeschäftigung seltener stattfinden als in Berufen mit einer schwächeren Ideal Worker Norm.

H2a: Je stärker die Ideal Worker Norm im Beruf ausgeprägt ist, desto geringer sind die Übergangsraten in Teilzeit.

Allerdings legt auch die Perspektive der Gendered Organizations mit Blick auf den Übergang in Teilzeitarbeit nahe, dass die beruflichen Arbeitszeitarrangements nicht für beide Geschlechter gleichermaßen wirkmächtig sind. Abgeleitet aus individuellen Geschlechterrollenzuschreibungen gelten Männer generell als ideale Arbeitnehmer, die der Ideal Worker Norm entsprechen (Acker 1990). Teilzeitarbeit stellt für Männer zusätzlich zur Abweichung von der Ideal Worker Norm auch eine Abweichung von der männlichen Geschlechterrolle dar und wird als unmännlich bewertet (Rudman und Mescher 2013; Vandello et al. 2013). Um dem zu entgehen, sollten vor allem Männer in Berufen mit einer starken Ideal Worker Norm besonders selten in Teilzeit übergehen.

Im Gegensatz dazu können Frauen aufgrund ihrer gesellschaftlich zugeschriebenen Zuständigkeit für Kindererziehung und Hausarbeit den Normen des idealen Arbeitsnehmers von vornherein nicht entsprechen (Acker 1990). Das bedeutet auch, dass eine Abweichung von der Ideal Worker Norm für Frauen und insbesondere für Mütter im Einklang mit ihrer Geschlechterrolle steht und daher akzeptierter ist oder 
auch teilweise erwartet wird (Rudman und Mescher 2013; Vandello et al. 2013). Unterstützt wird diese Abweichung in Deutschland durch das nach wie vor weit verbreitete modifizierte männliche Ernährermodell, demzufolge sich familienpolitische Maßnahmen immer noch am Ideal der ,good mother“ (Grunow et al. 2006, S. 123) ausrichten. Dadurch kommt es insbesondere für Frauen mit Careaufgaben in denjenigen Berufen zu Vereinbarkeitsproblemen, in denen Vollzeitarbeit, Mehrarbeit und Präsenz vor Ort die Norm darstellen. Teilzeitarbeit ist dann zwar eine Abweichung von den vorherrschenden Normen des idealen Arbeitnehmers, jedoch im Einklang mit der Norm der guten Mutter und kann dadurch für Frauen eine Lösung sein, um im Beruf zu verbleiben. Daher sollte die Ideal Worker Norm hier geschlechterdifferent wirken.

$H 2 b$ : Die Ideal Worker Norm in Berufen sollte für Männer einen stärkeren Einfluss auf den Übergang in Teilzeit haben als für Frauen.

\section{Daten, Operationalisierungen und Methode}

Für die empirischen Analysen verwenden wir Daten der Erwachsenenbefragung des Nationalen Bildungspanels (NEPS, Startkohorte 6) (Blossfeld et al. 2011). ${ }^{4}$ Diese Daten basieren auf einer repräsentativen Stichprobe von ca. 17.100 Erwachsenen der Geburtsjahrgänge 1944 bis 1986 in Deutschland, für die ausführliche retrospektive Angaben zu Erwerbsverläufen, Bildungsphasen, Arbeitslosigkeiten oder Erziehungszeiten erhoben wurden (Allmendinger et al. 2019). Wir untersuchen Männer und Frauen, die im Zeitraum zwischen 1992 und 2015 erwerbstätig waren, da vor diesem Zeitraum Männer in sehr geringem Maße Teilzeit gearbeitet haben. Zudem beschränken wir die Stichprobe auf abhängig Beschäftigte im Alter von 20 bis 55 Jahren mit erfolgtem Arbeitsmarkteinstieg, um Teilzeitepisoden während der Ausbildung und Altersteilzeitprogramme auszuschließen und dadurch den Fokus auf die Familienphase im Erwerbsverlauf zu legen. ${ }^{5}$

In unseren Analysen untersuchen wir die Dauer vom Beginn einer Vollzeiterwerbstätigkeit bis zu einem Wechsel in eine sozialversicherungspflichtige Teilzeittätigkeit. Geringfügige Beschäftigungsverhältnisse werden nicht berücksichtigt, da sich die Gründe zwischen diesen beiden Formen der Teilzeitarbeit deutlich unterscheiden (Brenke 2011), die NEPS-Daten aber keine Gründe für die Teilzeitbeschäftigung enthalten. In Anlehnung an empirische Analysen zur Teilzeitarbeit in Deutschland (z.B. Wanger 2015; Wolf 2014) definieren wir Teilzeittätigkeiten als

\footnotetext{
${ }^{4}$ Diese Arbeit nutzt Daten des Nationalen Bildungspanels (NEPS) Startkohorte 6 (Erwachsene), https:// doi.org/10.5157/NEPS:SC6:7.0.0. Die Daten des NEPS wurden von 2008 bis 2013 als Teil des Rahmenprogramms zur Förderung der empirischen Bildungsforschung erhoben, welches vom Bundesministerium für Bildung und Forschung (BMBF) finanziert wurde. Seit 2014 wird NEPS vom Leibniz-Institut für Bildungsverläufe e. V. (LIfBi) an der Otto-Friedrich-Universität Bamberg in Kooperation mit einem deutschlandweiten Netzwerk weitergeführt.

5 Als Arbeitsmarkteinstieg definieren wir die erste Erwerbstätigkeit nach der Berufsausbildung mit einer Arbeitszeit von mehr als 15 Wochenstunden und mit einer Mindestdauer von 6 Monaten (siehe Hägglund und Bächmann 2017 für eine ähnliche Definition).
} 
Erwerbstätigkeiten mit einem vertraglich vereinbarten Stundenumfang von mehr als 15 und weniger als 35 Wochenstunden. ${ }^{6}$ Tätigkeiten mit einem Stundenumfang von 35 Wochenstunden und mehr gelten als Vollzeit, solche mit 15 Wochenstunden und weniger als geringfügige Beschäftigung. ${ }^{7}$

Um eine vergleichbare Arbeitsmarktorientierung der Personen in der Stichprobe zu gewährleisten, ist der Ausgangspunkt für einen Wechsel in Teilzeitbeschäftigung immer eine Erwerbstätigkeit in Vollzeit. Damit beschränken wir uns auf Übergänge in Teilzeitbeschäftigung, die eine Reduzierung der Arbeitszeit darstellen. Allerdings finden Übergänge in Teilzeit sowohl für Frauen als auch für Männer häufig aus der Inaktivität, z. B. aus einer Arbeitslosigkeit oder Elternzeit, heraus statt (Brenke 2011). Da dies auch in unserer Stichprobe der Fall ist (siehe Anhang, Tab. 3), werden Erwerbslücken von bis zu 36 Monaten im Anschluss an eine Vollzeittätigkeit zugelassen, aus denen Personen noch in eine Teilzeitbeschäftigung wechseln können. ${ }^{8}$

In der finalen Analysestichprobe verbleiben 9058 Personen, davon 3911 Frauen und 5147 Männer, die mindestens einmal im Zeitraum von 1992 bis 2015 Vollzeit erwerbstätig waren. Insgesamt liegen 19.775 Episoden von Vollzeittätigkeiten vor, von denen $28 \%$ am Ende eine Erwerbslücke von bis zu 36 Monaten aufweisen. Um die unabhängigen Variablen und Kontrollvariablen als zeitveränderliche Merkmale in den Analysen zu berücksichtigen, haben wir ein Episodensplitting auf Monatsbasis vorgenommen (Blossfeld et al. 2007). Für Frauen liegen insgesamt 415.852 Analysemonate vor und wir beobachten 717 Übergänge in Teilzeittätigkeiten. Bei Männern liegen 672.892 Analysemonate und 263 Übergänge in Teilzeittätigkeiten vor.

Für die unabhängigen Variablen wurden zeitveränderliche Berufsangaben, basierend auf den Scientific Use Files des Mikrozensus für die Jahre 1993 und 1995 bis 2012, generiert. Da wir uns für die beruflichen Gelegenheitsstrukturen für Teilzeitarbeit im Ausgangsberuf interessieren, werden diese Berufsangaben den Vollzeittätigkeiten über die Berufsordnungen der Klassifikation der Berufe 1988 (3-Steller KldB88) und das Kalenderjahr als Merkmale hinzugespielt. Nach notwendigen Harmonisierungen der Berufscodierungen in den unterschiedlichen Wellen im Mikrozensus wurden Berufe mit einer Ähnlichkeit von nötigen Kenntnissen, ausgeübten Tätigkeiten, Techniken und erforderlichen Lizenzen zusammengefasst (Matthes et al. 2008). Insgesamt unterscheiden wir 177 verschiedene Berufe in unserem Analyse-

\footnotetext{
${ }^{6}$ In der Forschung sind sowohl 30 Wochenstunden (z. B. Hipp und Stuth 2013) als auch 35 Wochenstunden (z.B. Dieckhoff et al. 2016) als Grenze für die Trennung zwischen Teilzeit- und Vollzeitbeschäftigung etabliert. Wir wählen die Grenze von 35 Wochenstunden, um hier auch vollzeitnahe Teilzeitbeschäftigung zu berücksichtigen.

7 Bei parallelen Teilzeittätigkeiten mit insgesamt weniger als 35 Wochenstunden wird die erste begonnene Tätigkeit berücksichtigt, parallele Tätigkeiten mit 35 Wochenstunden und mehr werden als Vollzeitepisoden behandelt (ca. 10\% der Teilzeittätigkeiten).

8 Dies entspricht dem gesetzlichen Kündigungsschutz für die Dauer der Elternzeit von 36 Monaten, der für den gesamten Untersuchungszeitraum konstant ist (Bächmann und Gatermann 2017). Modelle nur für direkte Übergänge aus der Vollzeit- in die Teilzeitbeschäftigung zeigen von den Effekten her ähnliche Ergebnisse wie die Modelle mit Lücken, aufgrund der geringeren Anzahl an Übergängen jedoch auf geringerem Signifikanzniveau (nicht dargestellt, auf Anfrage von den Autorinnen erhältlich).
} 
sample. Die Berufsangaben aggregieren wir für erwerbstätige Personen im Alter von 15 bis 65 Jahren gewichtet und mit einer Besetzung von mindestens 30 Personen pro Beruf und Jahr. Die Berufsangaben für fehlende Jahre des Mikrozensus extrapolieren wir über exponentielle Glättung der vorhandenen Angaben. Für den gesamten Beobachtungszeitraum liegen somit vollständige Angaben für alle identifizierten Berufe vor (4248 Berufe-Jahre).

Die zentralen unabhängigen Variablen für unsere Analysen sind die berufliche Geschlechterkomposition sowie berufliche Arbeitszeitarrangements, die die Prävalenz der Ideal Worker Norm signalisieren. Die Geschlechterkomposition setzen wir mit der Kategorisierung über den Frauenanteil im Beruf um. Berufe werden in frauendominierte (Frauenanteil von $70 \%$ und mehr), männerdominierte (Frauenanteil von $30 \%$ und weniger) und gemischte (Frauenanteil von mehr als $30 \%$ und weniger als $70 \%$ ) Berufe eingeteilt (z. B. auch bei Bächmann und Gatermann 2017; Busch 2013). Die Prävalenz der Ideal Worker Norm operationalisieren wir entsprechend der drei identifizierten Verhaltenselemente über drei Arbeitszeitindikatoren. Erstens ermitteln wir den Anteil der Vollzeitbeschäftigten im Beruf. Zweitens messen wir den Anteil an Erwerbstätigen je Berufskategorie, die angeben, im Vergleich zu ihrer normalen Wochenarbeitszeit in der Berichtswoche oder in der letzten Woche mehr gearbeitet zu haben. Die Anwesenheitsnorm operationalisieren wir über den Anteil an Erwerbstätigen je Berufskategorie, die angeben, ihre Tätigkeit nie von zu Hause auszuüben. Für die Analysen teilen wir die Indikatoren zu Vollzeit und Mehrarbeit in Terzile ein, da beide Variablen sehr schiefe Verteilungen aufweisen und zudem keine etablierte Kategorisierung wie für die Geschlechterkomposition existiert. Anwesenheit im Beruf wurde für die deskriptiven Analysen ebenfalls in Terzile aufgeteilt, konnte in den multivariaten Analysen aufgrund der Verletzung der Proportionalitätsannahme des Gesamtmodells jedoch nur als metrisches Merkmal berücksichtigt werden. ${ }^{9}$ Eine Deskription der beruflichen Arbeitszeitmerkmale ist in Tab. 1 dargestellt, wobei die Terzileinteilungen geringe bis mittlere Assoziationen aufweisen (Spearman's Rho von $-0,04$ bis -0,45). Ein starker Zusammenhang findet sich nur zwischen der Geschlechterkomposition und dem Vollzeitanteil im Beruf (Spearman's Rho $=-0,81$ ), weswegen wir den Einfluss der Arbeitszeitmerkmale auch unabhängig von der Geschlechterkomposition modellieren. ${ }^{10}$

Kontrollvariablen auf der Individualebene beinhalten das Alter, die Staatsangehörigkeit und als Humankapital den höchsten beruflichen Bildungsabschluss und die Berufserfahrung, zudem, ob Personen vorangegangene Teilzeiterfahrung besitzen und ob die aktuelle Vollzeittätigkeit mit einem Berufswechsel endet. Mit Blick auf teilzeitrelevante Beschäftigungsmerkmale kontrollieren wir für Leitungsfunktionen, Arbeitsort in Ost- oder Westdeutschland, Betriebsgröße, Tätigkeiten im öffentlichen Dienst und das Vorliegen einer Erwerbslücke von maximal 36 Monaten. Des Weite-

\footnotetext{
9 Bislang wurden diese Merkmale primär als metrische Variablen untersucht (z. B. Busch 2013; Leuze und Strauß 2016). Um die Robustheit unserer Ergebnisse zu prüfen, haben wir die Arbeitszeitmerkmale zusätzlich als metrische Variablen und als Quintile berücksichtigt (siehe Anhang, Tab. 6). Während die metrischen Variablen keine signifikanten Effekte zeigen, verweisen wir in der Ergebnisdiskussion auf relevante Gemeinsamkeiten und Unterschiede der Quintil-Operationalisierung.

10 Die Ergebnisse mit und ohne Berücksichtigung der Geschlechterkomposition sind sehr ähnlich (vgl. Anhang, Tab. 4 und 5), weswegen die bestehende Multikollinearität toleriert werden kann.
} 
Tab. 1 Deskription der Berufsvariablen zu Arbeitszeitarrangements, Berufssample 1992 bis 2015. (Daten: Mikrozensus 1993-2012, eigene Berechnungen)

\begin{tabular}{llll}
\hline Variable & Vollzeitanteil im Beruf & $\begin{array}{l}\text { Anteil Mehrarbeit im } \\
\text { Beruf }\end{array}$ & $\begin{array}{l}\text { Anteil ohne Heimar- } \\
\text { beit im Beruf }\end{array}$ \\
\hline Arithm. Mittel & 82,19 & 14,33 & 86,01 \\
Standardabweichung & 16,20 & 6,25 & 16,15 \\
(SD) & & 0,00 & 16,01 \\
Minimum (Min) & 16,75 & 11,03 & 86,8 \\
1. Terzil & 78,58 & 13,32 & 92,46 \\
Median & 87,45 & 16,24 & 96,08 \\
2. Terzil & 93,21 & 50,29 & 100 \\
Maximum (Max) & 100 & &
\end{tabular}

$N=4248$

ren berücksichtigen wir den Partnerschaftsstatus, die Bildungsrelation im Paar sowie das Alter des jüngsten Kindes im Haushalt. Zusätzlich enthalten die Modelle Dummys für die Zeitperioden 1992 bis 2000, 2001 bis 2006 und 2007 bis 2015, um wichtige politische Veränderungen, wie die Einführung des Rechts auf Teilzeitarbeit (Teilzeit- und Befristungsgesetz in 2001) und die Hartz-Reformen (2002 bis 2003) sowie die Reform von Elterngeld und Elternzeit (Bundeselterngeld- und Elternzeitgesetz in 2007), zu berücksichtigen. ${ }^{11}$ Tab. 2 gibt einen deskriptiven Überblick über alle berïcksichtigten Variablen in unserer Analysestichprobe.

Für unsere Auswertungen verwenden wir Methoden der Ereignisdatenanalyse und schätzen die Übergangsrate (Hazardrate) für den Wechsel aus Vollzeit- in Teilzeittätigkeiten (Blossfeld et al. 2007). Ereignisdatenanalysen haben zum einen den Vorteil, dass die Zeit, die Personen im Ausgangszustand verbracht haben, mit in die Berechnung der Hazardrate mit einfließt. In unserem Fall bedeutet dies, dass eine unterschiedlich lange Dauer der Vollzeitbeschäftigung und ggf. anschließende Erwerbslücken in der Modellierung berücksichtigt werden. Zum anderen werden auch rechtszensierte Beobachtungen in der Modellierung kontrolliert, d.h. Personen, deren Vollzeittätigkeiten am Ende des Beobachtungszeitraums noch nicht abgeschlossen sind und die möglicherweise erst später in Teilzeitbeschäftigung wechseln.

Für die Schätzung der Hazardrate und des Einflusses der beruflichen Merkmale verwenden wir Cox-Regressionsmodelle auf Monatsbasis (Cleves 1999; Blossfeld et al. 2007). Wir schätzen die Modelle mit geclusterten Standardfehlern auf Personenebene, da Personen im Beobachtungszeitraum mehrmals eine Vollzeitbeschäftigung innehaben und entsprechend mehrere Übergänge in Teilzeitbeschäftigung aufweisen können (Cleves 1999). Um die Mehrebenenstruktur nach Berufen zu berücksichtigen, ist der KlbB88 3-Steller als Stratifizierungsvariable in den Modellen enthalten. Durch dieses Vorgehen unterscheidet sich die Baselinehazardrate zwischen Berufen, die Berufsmerkmale in unterschiedlichen Berufen haben aber denselben Effekt auf die Übergangsraten (König et al. 2014). Da für Frauen und Männer

\footnotetext{
${ }^{11}$ Sieben Kontrollvariablen weisen fehlende Werte im Umfang von weniger als $5 \%$ auf. Insgesamt werden durch listenweisen Fallausschluss 7\% der Personenmonate im ursprünglichen Sample ausgeschlossen.
} 


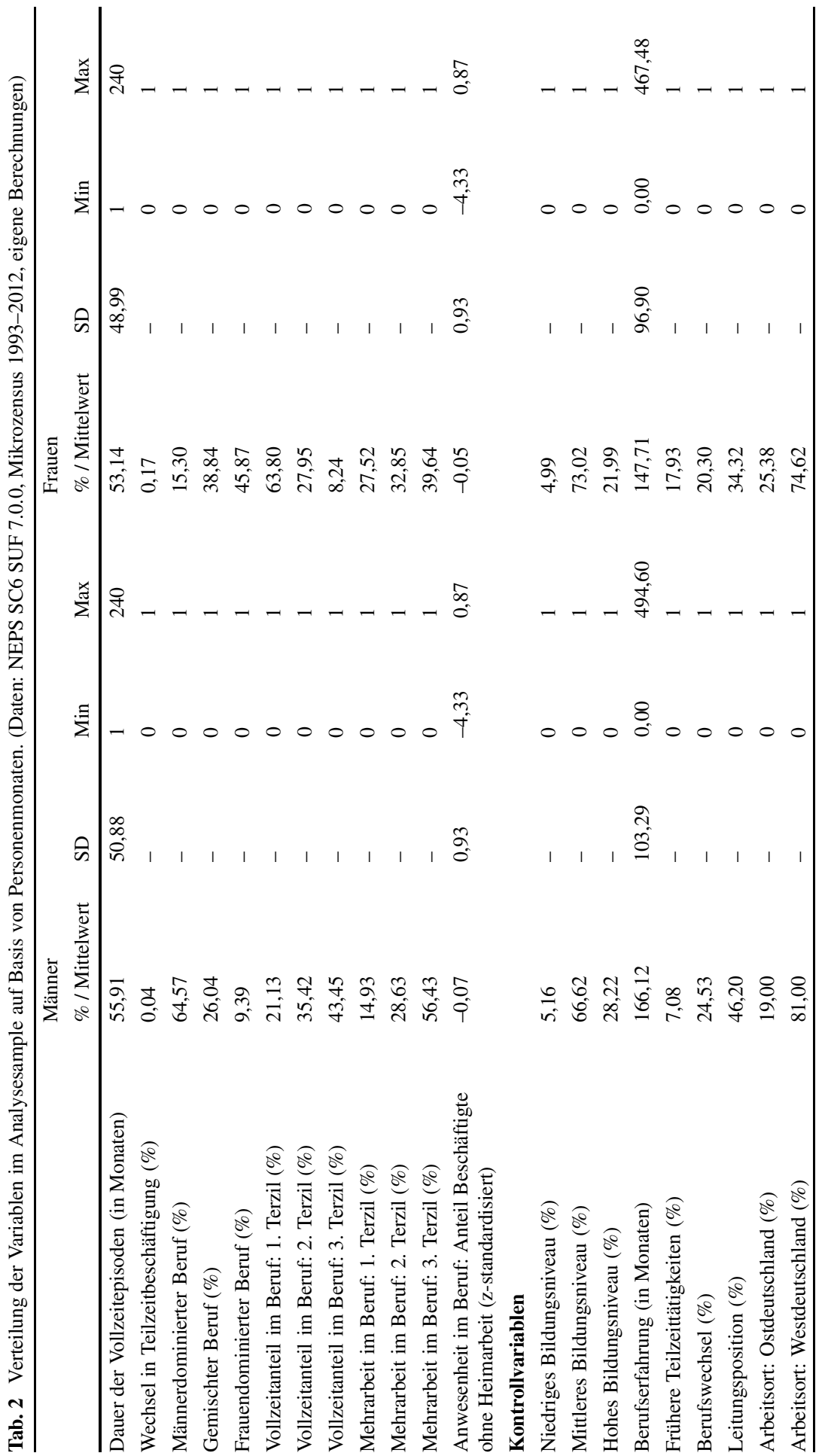




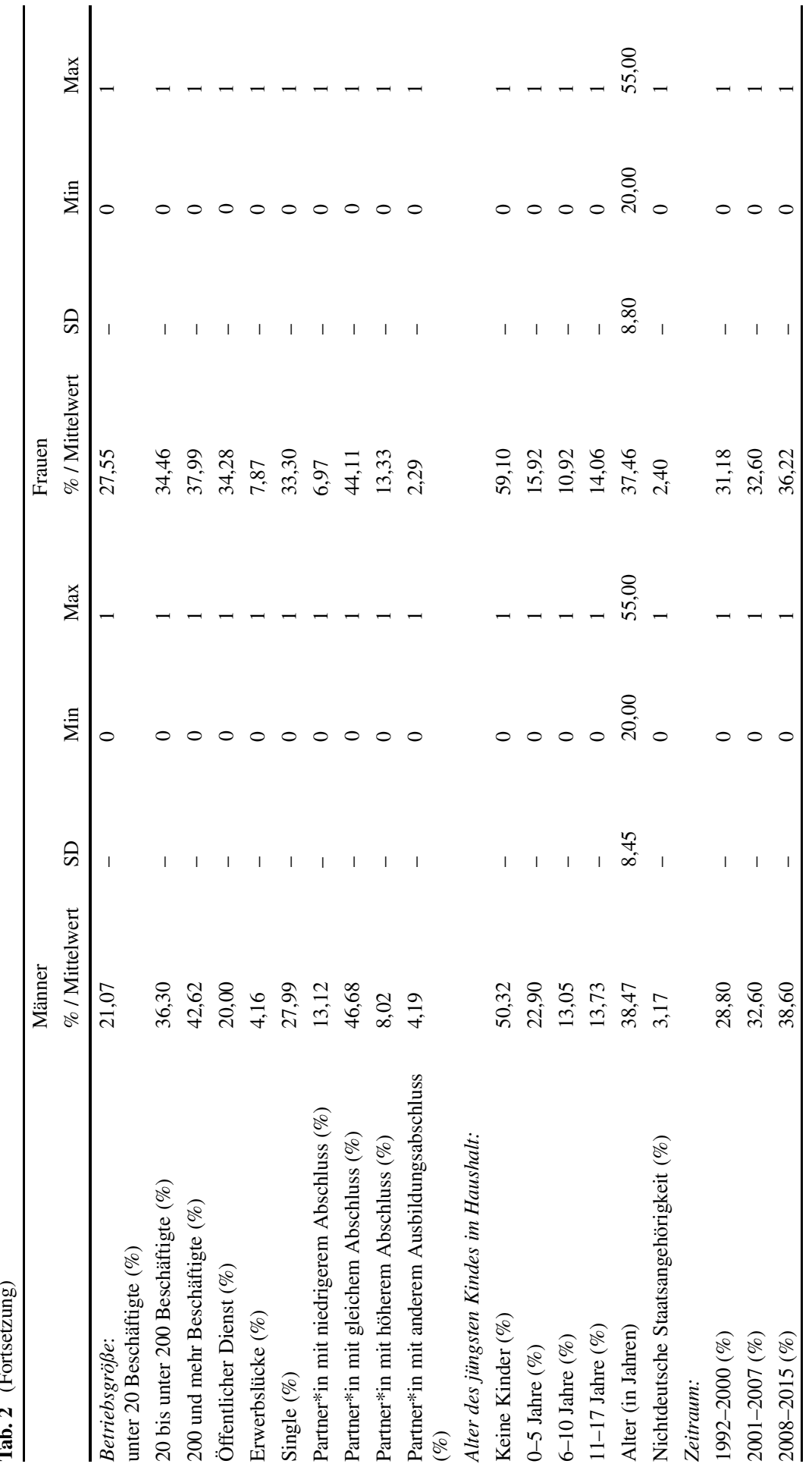


unterschiedliche Einflüsse der Berufsmerkmale vorliegen können, spezifizieren wir durchgehend getrennte Modelle für Frauen und Männer. ${ }^{12}$

\section{Ergebnisse}

Im Folgenden stellen wir die Ergebnisse unserer empirischen Analysen zur Bedeutung von beruflicher Geschlechtersegregation und beruflichen Arbeitszeitarrangements für den Übergang in Teilzeit vor. Wir untersuchen zunächst deskriptiv die Kaplan-Meier-Failure-Kurven von Männern und Frauen zum Übergang in Teilzeitbeschäftigung differenziert nach den unterschiedlichen Berufsmerkmalen (Abb. 1). Sie geben je Monat der Tätigkeitsdauer in Vollzeit die kumulierten Anteile an Personen an, die bis dahin einen Wechsel in eine Teilzeitbeschäftigung vollzogen haben. Erwartungsgemäß haben Männer in allen Grafiken deutlich geringere Übergangsraten in Teilzeit als Frauen. Mit Blick auf die Geschlechterkomposition in Berufen zeigt sich, dass sowohl Männer als auch Frauen häufiger aus frauendominierten und seltener aus männerdominierten Berufen in Teilzeit wechseln. Dies spricht von der Tendenz her für die Annahmen zur Institutionalisierung von Geschlechterrollen im Beruf, nämlich dass frauendominierte Berufe für beide Geschlechter einen leichteren Übergang in Teilzeit ermöglichen.

Für die drei Berufsmerkmale der Ideal Worker Norm finden wir unterschiedlich klare Ergebnisse. Mit Blick auf Vollzeitarbeit zeigt sich für beide Geschlechter, dass ein höherer Vollzeitanteil im Beruf mit geringeren Übergangsraten in Teilzeitbeschäftigung einhergeht. Die Kurven zur Mehrarbeit im Beruf sind dagegen weniger eindeutig. Von der Tendenz her scheinen Männer umso seltener in Teilzeit überzugehen, je mehr Überstunden im Beruf geleistet werden, während bei Frauen keine eindeutigen Tendenzen zu erkennen sind. Für den Anteil an Beschäftigten ohne Heimarbeit im Beruf lassen sich in den Kurven weder für Männer noch für Frauen klare Tendenzen erkennen. In Bezug auf unsere Annahmen der Ideal Worker Norm liefert unsere Deskription erste Hinweise darauf, dass Vollzeit und Mehrarbeit den Übergang in Teilzeit in der erwarteten Richtung strukturieren, zumindest bei Männern, dass jedoch nicht alle drei Verhaltenselemente gleichermaßen relevant sind.

Im nächsten Schritt untersuchen wir mithilfe von Cox Proportional Hazard-Modellen, inwiefern der Einfluss der Berufsmerkmale nach Kontrolle von Individualund Haushaltsmerkmalen zu beobachten ist. Abb. 2 stellt die Koeffizienten und die 95\%-Konfidenzintervalle der vier Berufsmerkmale aus den Modellen für Männer und Frauen grafisch dar. Werte über 0 signalisieren einen positiven Einfluss und damit eine höhere Übergangsrate in Teilzeit, Werte unter 0 einen negativen Einfluss auf die Übergangsrate. Die grauen Marker zeigen Koeffizienten aus Modellen,

\footnotetext{
12 Die Proportionalitätsannahme der Cox-Modelle haben wir mittels Schönfeld-Residuenanalyse überprüft. Diese zeigte eine Verletzung der Annahme für die Variablen Berufswechsel und Teilzeiterfahrung in den Frauenmodellen und für die Variablen Berufswechsel und Leitungstätigkeit in den Männermodellen. Für diese wurden daher Interaktionsterme mit der Verweildauer aufgenommen (siehe Blossfeld et al. 2007; Hägglund und Bächmann 2017 für das gleiche Vorgehen). Nach dieser Anpassung liegen keine weiteren Verletzungen der Proportionalitätsannahme vor.
} 

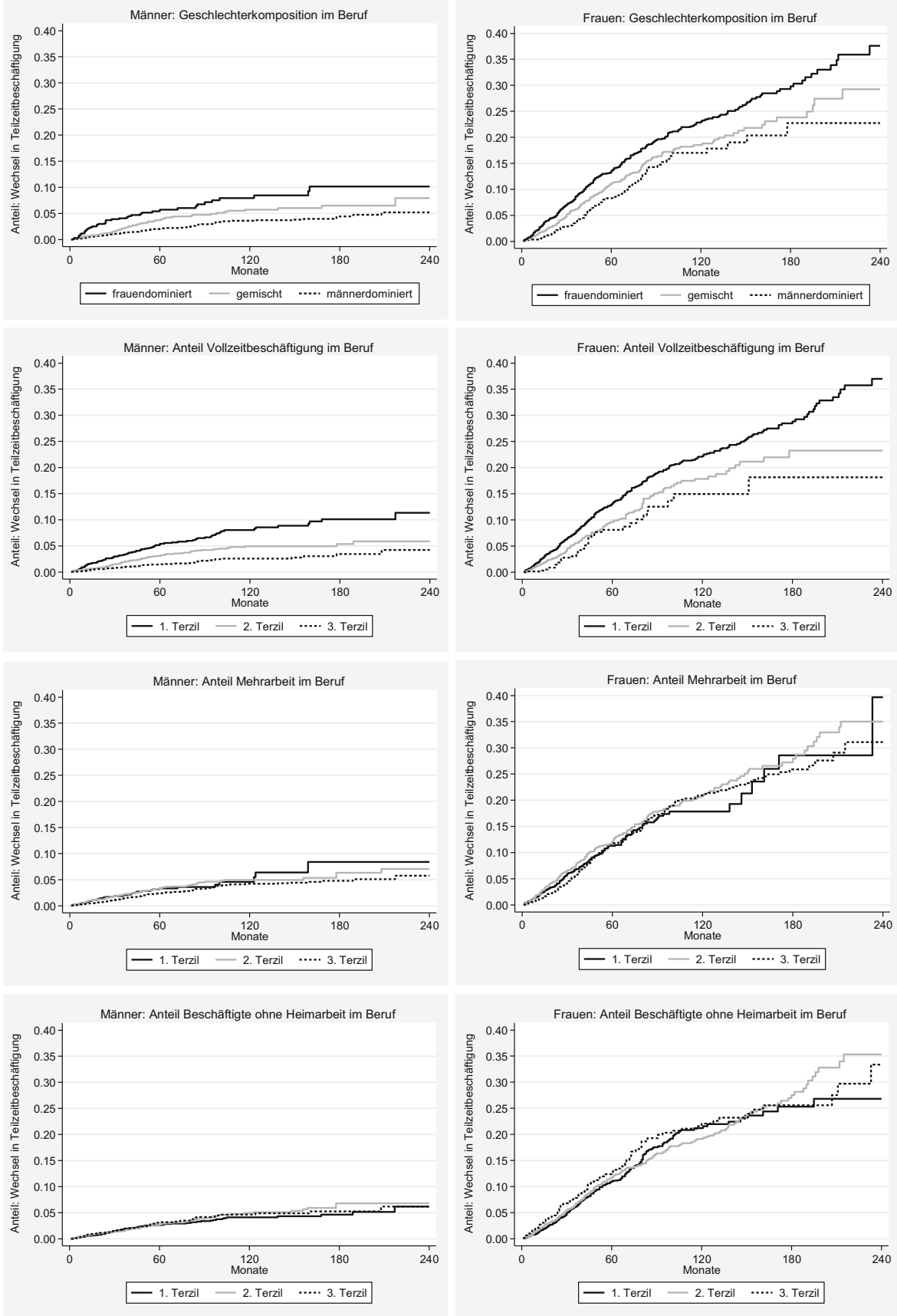

Abb. 1 Kaplan-Meier-Failure-Kurven für den Übergang in Teilzeit nach Geschlecht und Berufsmerkmalen. (Daten: NEPS SC6 SUF 7.0.0, Mikrozensus 1993-2012, eigene Berechnungen) 
Abb. 2 Cox-Proportional-Hazard-Modelle zum Übergang in Teilzeitbeschäftigung, Koeffizienten und $95 \%$-Konfidenzintervalle für Berufsmerkmale, Modelle kontrollieren für Variablen auf Individual- und Haushaltsebene. (Daten: NEPS SC6 SUF 7.0.0, Mikrozensus 1993-2012, eigene Berechnungen)

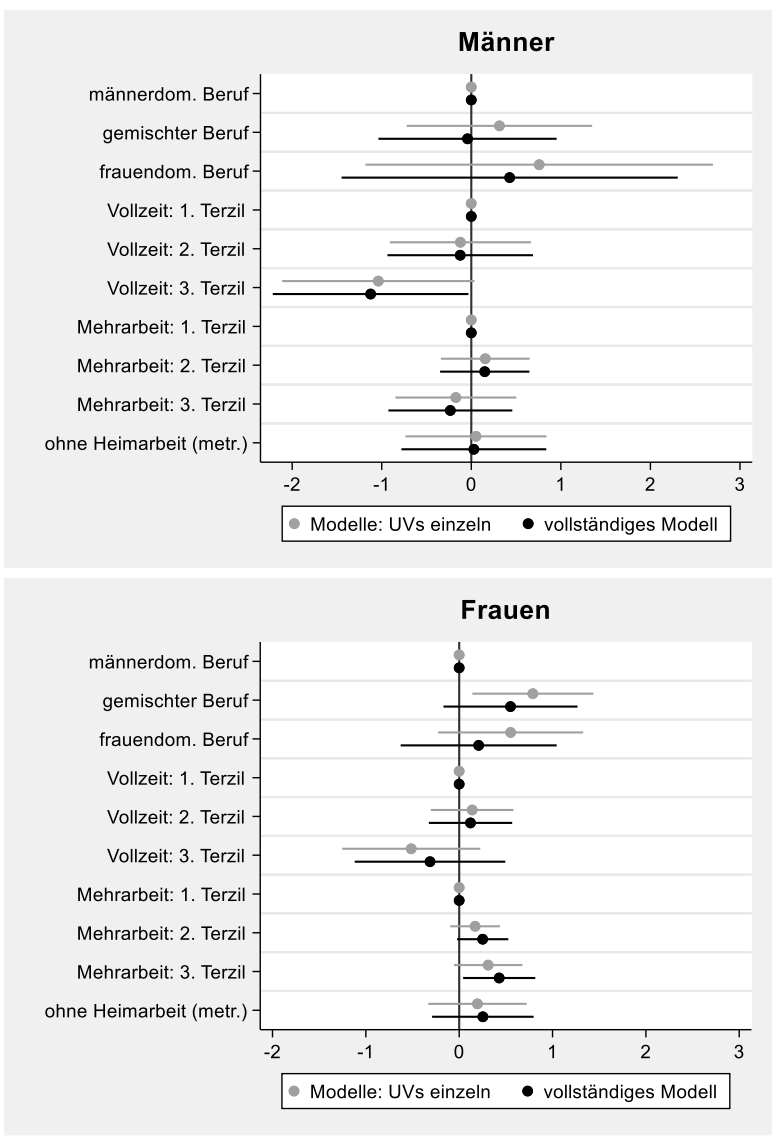

in denen die Berufsmerkmale jeweils einzeln aufgenommen wurden. Die schwarzen Marker bilden die Koeffizienten aus dem vollständigen Modell mit allen vier Berufsmerkmalen ab. ${ }^{13}$

In den multivariaten Analysen zeigt sich, dass die Geschlechterkomposition im Beruf weder für Männer noch für Frauen einen signifikanten Einfluss auf den Übergang in Teilzeitbeschäftigung hat. Von der Tendenz her scheinen Teilzeitübergänge für Männer häufiger aus frauendominierten Berufen zu erfolgen, während Frauen eher aus gemischten im Vergleich zu Männerberufen in Teilzeit wechseln. Insgesamt werden die Koeffizienten für die Geschlechterkomposition im Beruf unter Berücksichtigung der beruflichen Arbeitszeitarrangements geringer und nicht mehr signifikant, was vor allem auf den starken Zusammenhang mit dem Vollzeitanteil im Beruf zurückzuführen ist.

13 Die Tabellen für den schrittweisen Aufbau der Modelle (UVs einzeln) finden sich in Anhang, Tab. 4 und 5. Für die vollständigen Modelle sind im Anhang, Tab. 6, Modell M5 zusätzlich die Kontrollvariablen dargestellt. 
Wir schließen daraus, dass die Geschlechterkomposition in Berufen entgegen der Hypothese Hla und im Gegensatz zu den deskriptiven Befunden keinen eigenständigen Einfluss auf die Übergänge in Teilzeitbeschäftigung hat, weder für Männer noch für Frauen. Daher können wir auch Hypothese $H 1 b$ nicht beibehalten, wonach die berufliche Geschlechterkomposition für Frauen einen stärkeren Einfluss auf den Übergang in Teilzeit haben sollte als für Männer. Darüber hinaus weisen die Ergebnisse darauf hin, dass die Geschlechterkomposition nicht linear wirkt und dass die Übergangsraten in Teilzeitbeschäftigung in frauendominierten Berufen somit nicht am höchsten sein müssen. Dies könnte damit zusammen hängen, dass Teilzeitarbeit in frauendominierten Berufen zwar weit verbreitet ist, dafür aber Vollzeitstellen arbeitgeberseitig kaum mehr angeboten werden (Klenner und Lott 2016). Deswegen können wir hier auch kaum Übergänge aus Vollzeit in Teilzeit beobachten. Selbst wenn also in frauendominierten Berufen die Vereinbarkeit von Beruf und Familie auf beruflicher Ebene stärker institutionalisiert ist als in männerdominierten Berufen (Krüger 1995a), scheinen individuelle und haushaltsbezogene Einflussfaktoren sowie Arbeitszeitarrangements in Berufen relevanter für den Übergang in Teilzeit zu sein als die berufliche Geschlechterkomposition.

Für die drei Merkmale zu beruflichen Arbeitszeitarrangements, die die Ideal Worker Norm abbilden, finden wir in den multivariaten Analysen ebenso wie in der Deskription unterschiedlich klare Ergebnisse. So geht für Männer ein höherer Vollzeitanteil im Beruf mit geringeren Übergangsraten in Teilzeit einher: Arbeiten Männer in Berufen mit den höchsten Anteilen an Vollzeitbeschäftigten (3. Terzil), haben sie eine um ca. $68 \%$ geringere Übergangswahrscheinlichkeit in die Teilzeitbeschäftigung als in Berufen mit den geringsten Vollzeitanteilen $\left(1-\mathrm{e}^{-1,124}\right)$. Für Frauen hat der Anteil an Vollzeitbeschäftigten im Beruf dagegen keinen Einfluss auf die Übergangswahrscheinlichkeit in Teilzeitbeschäftigung.

Hinsichtlich der Verbreitung von Mehrarbeit im Beruf zeigen die Ergebnisse der Terzil-Operationalisierung für Männer keinen signifikanten Einfluss auf den Übergang in Teilzeit. Allerdings weist das Modell mit der Quintil-Operationalisierung für Männer für das 4. und 5. Quintil signifikant negative Koeffizienten aus (Anhang, Tab. 6, Modell M7). Dies spricht wiederum dafür, dass Berufe mit einem hohen Anteil an Mehrarbeit für Männer mit geringeren Übergangsraten in Teilzeitbeschäftigung einhergehen, zeigt jedoch auch, dass der Einfluss der Arbeitszeitmerkmale stark von der Operationalisierung abhängt. Für Frauen sehen wir im vollständigen Modell in Abb. 2 hingegen, dass sie umso häufiger in Teilzeit übergehen, je stärker Mehrarbeit im Beruf verbreitet ist. Damit haben Frauen in Berufen mit den höchsten Anteilen an Mehrarbeit eine um $54 \%$ höhere Übergangswahrscheinlichkeit $\left(1-\mathrm{e}^{0,429}\right)$ als Frauen in Berufen mit den niedrigsten Mehrarbeitsanteilen. Auch bei der QuintilOperationalisierung (Anhang, Tab. 6, Modell M7) hat das 4. Quintil für Frauen einen signifikanten und deutlich positiven Koeffizienten, was die Robustheit der Ergebnisse zur Mehrarbeit für Frauen in der Terzil-Operationalisierung stützt. Der Anteil an Beschäftigten ohne Heimarbeit hat in den multivariaten Analysen hingegen weder für Männer noch für Frauen einen Einfluss auf den Übergang in Teilzeit.

Insgesamt zeigen auch die multivariaten Analysen, dass nicht alle drei Arbeitszeitmerkmale und damit alle drei Verhaltenselemente der Ideal Worker Norm gleichermaßen für den Übergang in Teilzeit relevant sind. Vor allem die Vielarbeitsnorm, 
gemessen über den Vollzeitanteil und den Anteil an Mehrarbeit im Beruf, strukturiert den Übergang in Teilzeitarbeit, jedoch unterschiedlich für Männer und Frauen. So erschwert eine stärkere Prävalenz von Vollzeit- und Mehrarbeit den Übergang in Teilzeit vor allem für Männer, was für unsere Hypothese $H 2 b$ spricht. Als Ideal Worker Norm signalisieren ein hoher Vollzeit- und Überstundenanteil eine hohe Verfügbarkeitserwartung an die männlichen Beschäftigten in diesen Berufen. Aufgrund dessen wird ein Übergang in Teilzeit gerade in diesen Berufen vermieden. Da Teilzeitarbeit für Männer zusätzlich eine Abweichung von der männlichen Geschlechterrolle darstellt, verstärken individuelle Geschlechterrollenzuschreibungen möglicherweise diesen Verhinderungseffekt in Berufen mit einer starken Ideal Worker Norm.

Die Arbeitszeitmerkmale im Beruf wirken sich auch für Frauen auf die Übergänge in Teilzeitarbeit aus, jedoch nicht wie anhand der Ideal Worker Norm vermutet wurde. So finden wir für Frauen, dass ein höherer Anteil an Mehrarbeit im Beruf mit einer höheren Übergangswahrscheinlichkeit in Teilzeit assoziiert ist, was gegen die generelle Gültigkeit unserer Hypothese $H 2 a$ spricht. Offensichtlich können Frauen aufgrund ihrer gesellschaftlich zugeschriebenen Zuständigkeit für Kindererziehung und Hausarbeit der Norm des idealen Arbeitsnehmers von vornherein nicht entsprechen (Acker 1990). Stattdessen scheinen Frauen, gerade in Berufen mit einer ausgeprägten Überstundenkultur, eine Vereinbarkeit von Beruf und Familie dadurch zu erreichen, dass sie von diesen Normen abweichen und verstärkt in Teilzeit arbeiten. Für beide Geschlechter haben Anforderungen an die physische Präsenz am Arbeitsplatz dagegen keinen Einfluss auf den Übergang in Teilzeit.

\section{Diskussion}

In Deutschland bestehen ausgeprägte Geschlechterunterschiede in der Teilzeitbeschäftigung, was eine wesentliche Erklärung für weitere Geschlechterungleichheiten am Arbeitsmarkt ist. Neben individuellen und haushaltsbezogenen Erklärungsfaktoren gibt es Hinweise in der Literatur, dass strukturelle Gegebenheiten im Arbeitsmarkt, wie z.B. Berufsmerkmale, die Teilzeitbeschäftigung von Frauen und Männern beeinflussen können. Daher haben wir die Bedeutung der beruflichen Geschlechterkomposition und Arbeitszeitarrangements für den Übergang in Teilzeitarbeit untersucht. Anhand Helga Krügers Institutionenansatz zu geschlechtertypischen Lebensverläufen (1995a, 1995b, 2001) und Joan Ackers Ansatz der Gendered Organisations (1990) haben wir geschlechterdifferente Annahmen entwickelt. Denen zufolge sollte die Geschlechterkomposition in Berufen vor allem Übergänge in die Teilzeitarbeit von Frauen beeinflussen. Die beruflichen Arbeitszeitarrangements sollten dagegen vor allem auf die Teilzeitübergänge von Männern einen Einfluss haben.

Unsere empirischen Analysen zeigen erstens, dass sich die Geschlechterkomposition in Berufen an sich weder für Frauen noch für Männer auf den Übergang in die Teilzeitbeschäftigung auswirkt. Dass die Geschlechterkomposition keinen Einfluss auf Teilzeitübergänge hat, widerlegt jedoch nicht die Institutionalisierung von Geschlechterrollen in Berufen und Berufsstrukturen. Vielmehr verdeutlicht der Befund, dass die Geschlechterkomposition das Konzept der Nähe oder Ferne zu männlichen 
Berufskonstrukten nicht ausreichend abbildet. Zweitens strukturieren die untersuchten Arbeitszeitarrangements in Berufen die Übergänge in Teilzeitbeschäftigung, und zwar unabhängig von der Geschlechterkomposition in Berufen. Allerdings beeinflussen berufliche Arbeitszeitarrangements Männer und Frauen in entgegengesetzter Weise. So stützen unsere Ergebnisse nur für Männer die Erwartungen aus dem Ansatz der Gendered Organizations, nämlich dass eine stark ausgeprägte Ideal Worker Norm in Berufen Übergänge in Teilzeitarbeit verhindert. Für die Teilzeitarbeit von Frauen scheinen diese Erwartungen an den idealen Arbeitnehmer nicht zu gelten. Dies deutet darauf hin, dass bei Teilzeitarbeit von Frauen die individuellen Geschlechterrollen, die ihnen die Hauptzuständigkeit für Haushalt und Familie zuschreiben, wirkmächtiger sind als berufliche Gelegenheitsstrukturen für Teilzeitarbeit. Gestützt wird dies durch den Wohlfahrtsstaat in Deutschland, der zumindest in Teilen weiterhin das männliche Ernährermodell und das Ideal der guten Mutter reproduziert (Trappe et al. 2015; Grunow et al. 2006).

Aus den erläuterten Zusammenhängen lassen sich verschiedene Schlussfolgerungen ziehen. So sind individuelle und haushaltsbezogene Faktoren, wie die Anforderungen durch Elternschaft an die Vereinbarkeit von Beruf und Familie oder persönliche Arbeitszeitwünsche zwar wichtige, aber für sich genommen keine ausreichenden Erklärungen für Teilzeitbeschäftigung. Vielmehr bestimmen auch strukturelle Bedingungen im Arbeitsmarkt, allen voran die Arbeitszeitarrangements in Berufen, die Teilzeitbeschäftigung wesentlich mit. Daher sollte künftige Forschung diese strukturellen Rahmenbedingungen in empirischen Analysen stärker als bisher berücksichtigen, besonders für die Erklärung der Teilzeitbeschäftigung von Männern. Hier zeigen unsere Ergebnisse jedoch auch, dass die Effekte von Arbeitszeitmerkmalen stark von der gewählten Operationalisierung abhängen, da bislang keine etablierten Konventionen für deren Messung bestehen.

Aufgrund der großen Heterogenität in der Teilzeitbeschäftigung von Frauen und Männern haben wir für unsere empirischen Analysen Designentscheidungen getroffen, um eine möglichst hohe Vergleichbarkeit der Übergänge in Teilzeitbeschäftigung für beide Geschlechter zu gewährleisten. Hieraus ergeben sich auch Limitationen unserer Analysen. So können unsere Schlussfolgerungen beispielsweise nicht auf andere Arten von Übergängen in Teilzeitarbeit und auf geringfügige Beschäftigung übertragen werden. Ausgehend von der hohen beruflichen Stabilität von Erwerbsverläufen in Deutschland haben wir uns darüber hinaus auf die Berufsmerkmale des Ausgangsberufs konzentriert. Auf mögliche Einflüsse von Berufsmerkmalen des neuen Berufs, der nach einem Wechsel aufgenommen wird, konnten wir an dieser Stelle ebenso wenig eingehen wie auf unterschiedliche Prozesse bei Übergängen mit und ohne Berufswechsel. Schließlich haben wir in unseren Analysen nur den Übergang in Teilzeit untersucht, nicht die Dauer der Teilzeitbeschäftigung und daran anschließende Verläufe. Wie die bisherige Forschung jedoch zeigt, leisten die Konsequenzen der Teilzeitarbeit vor allem dann einen klaren Beitrag zur Reproduktion von Geschlechterungleichheiten im Arbeitsmarkt, wenn sie über einen längeren Zeitraum ausgeübt wird. Daher sollte künftige Forschung zu Geschlechterungleichheiten die langfristigen Konsequenzen von Teilzeitarbeit in den Erwerbsverläufen von Frauen und Männern und deren berufliche Strukturierung in den Blick nehmen. 
Mit Blick auf politische Implikationen zeigen unsere Analysen, dass die Arbeitsorganisation in Berufen ein wichtiger Ansatzpunkt sein kann, um künftig mehr Männern oder Vätern die Nutzung von Teilzeitarbeit zu ermöglichen. So sollten insbesondere in Berufen mit einer starken Ideal Worker Norm Lösungen zur besseren Vereinbarkeit von Beruf und Familie geschaffen werden. Doch auch die Arbeitsmarkt- und Sozialpolitik trägt eine entsprechende Verantwortung an den bestehenden Geschlechterunterschieden in der Teilzeitarbeit. Sie kann politische Rahmenbedingungen schaffen, die weniger den Leitbildern der traditionellen Arbeitsteilung von Frauen und Männern folgen. Wichtige Ansatzpunkte sind daher weiterhin der Ausbau der Kinderbetreuung, eine Verringerung der geschlechtlichen Lohnlücke sowie eine Anhebung der durchschnittlich geringeren Löhne in frauendominierten Berufen.

Danksagung Wir danken den Teilnehmer*innen der Konferenz Occupations and Social Inequality am IAB im Juni 2017, der Tagung der DGS-Sektion Soziale Ungleichheit und Sozialstrukturanalyse im Mai 2019 sowie des AAM/NEPS/HSI-Kolloquiums am WZB für hilfreiche Diskussionen des Beitrags. Den Herausgeber*innen des Sonderbandes und den anonymen Gutachter*innen gilt unser Dank für ihre wertvollen und konstruktiven Hinweise.

Funding Open Access funding provided by Projekt DEAL.

Open Access Dieser Artikel wird unter der Creative Commons Namensnennung 4.0 International Lizenz veröffentlicht, welche die Nutzung, Vervielfältigung, Bearbeitung, Verbreitung und Wiedergabe in jeglichem Medium und Format erlaubt, sofern Sie den/die ursprünglichen Autor(en) und die Quelle ordnungsgemäß nennen, einen Link zur Creative Commons Lizenz beifügen und angeben, ob Änderungen vorgenommen wurden.

Die in diesem Artikel enthaltenen Bilder und sonstiges Drittmaterial unterliegen ebenfalls der genannten Creative Commons Lizenz, sofern sich aus der Abbildungslegende nichts anderes ergibt. Sofern das betreffende Material nicht unter der genannten Creative Commons Lizenz steht und die betreffende Handlung nicht nach gesetzlichen Vorschriften erlaubt ist, ist für die oben aufgeführten Weiterverwendungen des Materials die Einwilligung des jeweiligen Rechteinhabers einzuholen.

Weitere Details zur Lizenz entnehmen Sie bitte der Lizenzinformation auf http://creativecommons.org/ licenses/by/4.0/deed.de. 


\section{Anhang}

Tab. 3 Übergangsmatrix Teilzeit: relative Häufigkeiten von Übergängen in die Teilzeitbeschäftigung in der gesamten Stichprobe an Episoden von Männern und Frauen im Zeitraum 1992 bis 2015. (Daten: NEPS SC6 SUF 7.0.0, eigene Berechnungen)

\begin{tabular}{|c|c|c|c|}
\hline \multirow{3}{*}{$\begin{array}{l}\text { Art der Ausgangsepisode } \rightarrow \\
\text { Art der Anschlussepisode }\end{array}$} & \multicolumn{3}{|c|}{ Geschlecht } \\
\hline & Alle & Frauen & Männer \\
\hline & $\%$ & $\%$ & $\%$ \\
\hline Vollzeit $\rightarrow$ Teilzeit & 15,2 & 12,2 & 29,2 \\
\hline Inaktivität $\rightarrow$ Teilzeit & 50,1 & 51,9 & 41,6 \\
\hline Teilzeit $\rightarrow$ Teilzeit & 19,2 & 20,8 & 12,0 \\
\hline Geringfügige Beschäftigung $\rightarrow$ Teilzeit & 7,4 & 7,8 & 6,0 \\
\hline Parallele Teilzeit $>35 \mathrm{~h} \rightarrow$ Teilzeit & 8,0 & 7,4 & 11,1 \\
\hline Gesamt & 100 & 100 & 100 \\
\hline $\begin{array}{l}\text { Anteil Übergänge in die Teilzeitbeschäftigung an allen } \\
\text { Episodenwechseln }\end{array}$ & 10,6 & 16,0 & 4,2 \\
\hline Anzahl aller Episodenwechsel & $n=40.488$ & $n=22.043$ & $n=18.445$ \\
\hline Anzahl Personen & $n=9714$ & $n=5075$ & $n=4639$ \\
\hline
\end{tabular}


Tab. 4 Cox Proportional Hazard-Modelle zum Übergang in Teilzeitbeschäftigung für Männer, schrittweiser Modellaufbau. (Daten: NEPS SC6 SUF 7.0.0, Mikrozensus 1993-2012, eigene Berechnungen)

\begin{tabular}{|c|c|c|c|c|c|}
\hline & $\begin{array}{l}\text { Männer } \\
\text { M1 }\end{array}$ & $\begin{array}{l}\text { Männer } \\
\text { M2 }\end{array}$ & $\begin{array}{l}\text { Männer } \\
\text { M3 }\end{array}$ & $\begin{array}{l}\text { Männer } \\
\text { M4 }\end{array}$ & $\begin{array}{l}\text { Männer } \\
\text { M5 }\end{array}$ \\
\hline \multicolumn{6}{|c|}{ Geschlechterkomposition im Beruf (Ref.: Männerdom. Beruf) } \\
\hline \multirow[t]{2}{*}{ Gemischter Beruf } & 0,315 & - & - & - & $-0,042$ \\
\hline & $(0,528)$ & - & - & - & $(0,507)$ \\
\hline \multirow[t]{2}{*}{ Frauendom. Beruf } & 0,759 & - & - & - & 0,428 \\
\hline & $(0,990)$ & - & - & - & $(0,958)$ \\
\hline \multicolumn{6}{|c|}{ Vollzeitanteil im Beruf (Ref.: 1. Terzil) } \\
\hline \multirow[t]{2}{*}{ 2. Terzil } & - & $-0,121$ & - & - & $-0,124$ \\
\hline & - & $(0,402)$ & - & - & $(0,415)$ \\
\hline \multirow[t]{2}{*}{ 3. Terzil } & - & $-1,038+$ & - & - & $-1,124 *$ \\
\hline & - & $(0,548)$ & - & - & $(0,557)$ \\
\hline \multicolumn{6}{|c|}{ Anteil Mehrarbeit im Beruf(Ref.: 1. Terzil) } \\
\hline \multirow[t]{2}{*}{ 2. Terzil } & - & - & 0,156 & - & 0,150 \\
\hline & - & - & $(0,253)$ & - & $(0,254)$ \\
\hline \multirow[t]{2}{*}{ 3. Terzil } & - & - & $-0,172$ & - & $-0,234$ \\
\hline & - & - & $(0,344)$ & - & $(0,353)$ \\
\hline Anteil ohne Heimarbeit im & - & - & - & 0,052 & 0,029 \\
\hline $\begin{array}{l}\text { Beruf } \\
\text { (metrisch, z-standardisiert) }\end{array}$ & - & - & - & $(0,401)$ & $(0,413)$ \\
\hline$N$ Monate & 672.892 & 672.892 & 672.892 & 672.892 & 672.892 \\
\hline$N$ Episoden & 12.061 & 12.061 & 12.061 & 12.061 & 12.061 \\
\hline$N$ Übergänge & 263 & 263 & 263 & 263 & 263 \\
\hline$N$ Personen & 5147 & 5147 & 5147 & 5147 & 5147 \\
\hline Log Likelihood & $-785,056$ & $-782,571$ & $-784,393$ & $-785,435$ & $-780,968$ \\
\hline AIC & 1624,111 & 1619,143 & 1622,786 & 1622,869 & 1625,936 \\
\hline $\mathrm{Chi}^{2}$ & 563,836 & 605,888 & 573,332 & 564,271 & 626,745 \\
\hline Pseudo $\mathrm{R}^{2}$ & 0,255 & 0,258 & 0,256 & 0,255 & 0,259 \\
\hline
\end{tabular}

Koeffizienten, robuste Standardfehler in Klammern, Modelle kontrollieren für Variablen auf Individualund Haushaltsebene (für vollständiges Modell M5 inkl. Kontrollvariablen siehe Anhang, Tab. 6) ${ }^{+} p<0,1,{ }^{*} p<0,05,{ }^{* *} p<0,01,{ }^{* * * *} p<0,001$ 
Tab. 5 Cox Proportional Hazard-Modelle zum Übergang in Teilzeitbeschäftigung für Frauen, schrittweiser Modellaufbau. (Daten: NEPS SC6 SUF 7.0.0, Mikrozensus 1993-2012, eigene Berechnungen)

\begin{tabular}{|c|c|c|c|c|c|}
\hline & $\begin{array}{l}\text { Frauen } \\
\text { M1 }\end{array}$ & $\begin{array}{l}\text { Frauen } \\
\text { M2 }\end{array}$ & $\begin{array}{l}\text { Frauen } \\
\text { M3 }\end{array}$ & $\begin{array}{l}\text { Frauen } \\
\text { M4 }\end{array}$ & $\begin{array}{l}\text { Frauen } \\
\text { M5 }\end{array}$ \\
\hline \multicolumn{6}{|c|}{$\overline{\text { Geschlechterkomposition im Beruf (Ref.: Männerdom. Beruf) }}$} \\
\hline \multirow[t]{2}{*}{ Gemischter Beruf } & $0,789 *$ & - & - & - & 0,550 \\
\hline & $(0,331)$ & - & - & - & $(0,366)$ \\
\hline \multirow[t]{2}{*}{ Frauendom. Beruf } & 0,551 & - & - & - & 0,209 \\
\hline & $(0,396)$ & - & - & - & $(0,426)$ \\
\hline \multicolumn{6}{|c|}{ Vollzeitanteil im Beruf(Ref.: 1. Terzil) } \\
\hline \multirow[t]{2}{*}{ 2. Terzil } & - & 0,139 & - & - & 0,121 \\
\hline & - & $(0,226)$ & - & - & $(0,228)$ \\
\hline \multirow[t]{2}{*}{ 3. Terzil } & - & $-0,514$ & - & - & $-0,313$ \\
\hline & - & $(0,377)$ & - & - & $(0,411)$ \\
\hline \multicolumn{6}{|c|}{ Anteil Mehrarbeit im Beruf(Ref.: 1. Terzil) } \\
\hline \multirow[t]{2}{*}{ 2. Terzil } & - & - & 0,171 & - & $0,251+$ \\
\hline & - & - & $(0,136)$ & - & $(0,140)$ \\
\hline \multirow[t]{2}{*}{ 3. Terzil } & - & - & $0,311+$ & - & $0,429 *$ \\
\hline & - & - & $(0,187)$ & - & $(0,197)$ \\
\hline Anteil ohne Heimarbeit im & - & - & - & 0,195 & 0,254 \\
\hline $\begin{array}{l}\text { Beruf } \\
\text { (metrisch, z-standardisiert) }\end{array}$ & - & - & - & $(0,269)$ & $(0,278)$ \\
\hline$N$ Monate & 415.852 & 415.852 & 415.852 & 415.852 & 415.852 \\
\hline$N$ Episoden & 7714 & 7714 & 7714 & 7714 & 7714 \\
\hline$N$ Übergänge & 717 & 717 & 717 & 717 & 717 \\
\hline$N$ Personen & 3911 & 3911 & 3911 & 3911 & 3911 \\
\hline Log Likelihood & $-2455,676$ & $-2456,362$ & $-2456,954$ & $-2457,937$ & $-2452,827$ \\
\hline$A I C$ & 4965,353 & 4966,724 & 4967,908 & 4967,874 & 4969,654 \\
\hline $\mathrm{Chi}^{2}$ & 1501,087 & 1508,477 & 1476,858 & 1492,160 & 1502,460 \\
\hline Pseudo $\mathrm{R}^{2}$ & 0,210 & 0,209 & 0,209 & 0,209 & 0,211 \\
\hline
\end{tabular}

Koeffizienten, robuste Standardfehler in Klammern, Modelle kontrollieren für Variablen auf Individualund Haushaltsebene (für vollständiges Modell M5 inkl. Kontrollvariablen siehe Anhang, Tab. 6) ${ }^{+} p<0,1,{ }^{*} p<0,05,{ }^{* *} p<0,01,{ }^{* * *} p<0,001$ 
Tab. 6 Vollständige Modelle für alternative Operationalisierungen der Arbeitszeitarrangements in Berufen, Cox Proportional Hazard-Modelle zum Übergang in Teilzeitbeschäftigung. (Daten: NEPS SC6 SUF 7.0.0, Mikrozensus 1993-2012, eigene Berechnungen)

\begin{tabular}{|c|c|c|c|c|c|c|}
\hline & \multicolumn{2}{|c|}{$\begin{array}{l}\text { Operationalisierung als } \\
\text { TERZILE }\end{array}$} & \multicolumn{2}{|c|}{$\begin{array}{l}\text { METRISCHE } \\
\text { Operationalisierung }\end{array}$} & \multicolumn{2}{|c|}{$\begin{array}{l}\text { Operationalisierung als } \\
\text { QUINTILE }\end{array}$} \\
\hline & $\begin{array}{l}\text { Männer } \\
\text { M5 }\end{array}$ & $\begin{array}{l}\text { Frauen } \\
\text { M5 }\end{array}$ & $\begin{array}{l}\text { Männer } \\
\text { M6 }\end{array}$ & $\begin{array}{l}\text { Frauen } \\
\text { M6 }\end{array}$ & $\begin{array}{l}\text { Männer } \\
\text { M7 }\end{array}$ & $\begin{array}{l}\text { Frauen } \\
\text { M7 }\end{array}$ \\
\hline \multicolumn{7}{|c|}{ Berufsvariablen: metrisch } \\
\hline \multirow{2}{*}{$\begin{array}{l}\text { Männeranteil im } \\
\text { Beruf (z-stand.) }\end{array}$} & - & - & 0,253 & 0,465 & - & - \\
\hline & - & - & $(0,943)$ & $(0,558)$ & - & - \\
\hline \multirow{2}{*}{$\begin{array}{l}\text { Vollzeitanteil im } \\
\text { Beruf (z-stand.) }\end{array}$} & - & - & $-0,100$ & $-0,476+$ & - & - \\
\hline & - & - & $(0,443)$ & $(0,266)$ & - & - \\
\hline \multirow{2}{*}{$\begin{array}{l}\text { Anteil Mehrarbeit } \\
\text { im Beruf (z-stand.) }\end{array}$} & - & - & $-0,119$ & $-0,003$ & - & - \\
\hline & - & - & $(0,172)$ & $(0,107)$ & - & - \\
\hline \multirow{2}{*}{\multicolumn{2}{|c|}{$\begin{array}{lc}\text { Anteil ohne } & 0,029 \\
\text { Heimarbeit im Beruf } & (0,413) \\
\text { (z-stand.) } & \text { Berufsvariablen: kategorial }\end{array}$}} & 0,254 & $-0,023$ & 0,223 & - & - \\
\hline & & $(0,278)$ & $(0,447)$ & $(0,311)$ & - & - \\
\hline \multicolumn{7}{|c|}{ Geschlechterkomposition im Beruf (Ref.: Männerdom. Beruf) } \\
\hline \multirow[t]{2}{*}{ Gemischter Beruf } & $-0,042$ & 0,550 & - & - & 0,160 & $0,732 *$ \\
\hline & $(0,507)$ & $(0,366)$ & - & - & $(0,546)$ & $(0,332)$ \\
\hline \multirow[t]{2}{*}{ Frauendom. Beruf } & 0,428 & 0,209 & - & - & 0,374 & 0,406 \\
\hline & $(0,958)$ & $(0,426)$ & - & - & $(0,945)$ & $(0,411)$ \\
\hline \multicolumn{7}{|c|}{ Vollzeitanteil im Beruf(Ref.: 1. Terzil) } \\
\hline \multirow[t]{2}{*}{ 2. Terzil } & $-0,124$ & 0,121 & - & - & - & - \\
\hline & $(0,415)$ & $(0,228)$ & - & - & - & - \\
\hline \multirow[t]{2}{*}{ 3. Terzil } & $-1,124 *$ & $-0,313$ & - & - & - & - \\
\hline & $(0,557)$ & $(0,411)$ & - & - & - & - \\
\hline \multicolumn{7}{|c|}{ Anteil Mehrarbeit im Beruf (Ref.: 1. Terzil) } \\
\hline \multirow[t]{2}{*}{ 2. Terzil } & 0,150 & $0,251+$ & - & - & - & - \\
\hline & $(0,254)$ & $(0,140)$ & - & - & - & - \\
\hline \multirow[t]{2}{*}{ 3. Terzil } & $-0,234$ & $0,429^{*}$ & - & - & - & - \\
\hline & $(0,353)$ & $(0,197)$ & - & - & - & - \\
\hline \multicolumn{7}{|c|}{ Vollzeitanteil im Beruf(Ref.: 1. Quintil) } \\
\hline \multirow[t]{2}{*}{ 2. Quintil } & - & - & - & - & $-0,698$ & 0,118 \\
\hline & - & - & - & - & $(0,510)$ & $(0,229)$ \\
\hline \multirow[t]{2}{*}{ 3. Quintil } & - & - & - & - & $-0,173$ & $0,661 *$ \\
\hline & - & - & - & - & $(0,689)$ & $(0,335)$ \\
\hline \multirow[t]{2}{*}{ 4. Quintil } & - & - & - & - & $-0,770$ & 0,062 \\
\hline & - & - & - & - & $(0,763)$ & $(0,495)$ \\
\hline \multirow[t]{2}{*}{ 5. Quintil } & - & - & - & - & $-0,916$ & 0,375 \\
\hline & - & - & - & - & $(0,856)$ & $(0,754)$ \\
\hline
\end{tabular}


Tab. 6 (Fortsetzung)

\begin{tabular}{|c|c|c|c|c|c|c|}
\hline & \multicolumn{2}{|c|}{$\begin{array}{l}\text { Operationalisierung als } \\
\text { TERZILE }\end{array}$} & \multicolumn{2}{|c|}{$\begin{array}{l}\text { METRISCHE } \\
\text { Operationalisierung }\end{array}$} & \multicolumn{2}{|c|}{$\begin{array}{l}\text { Operationalisierung als } \\
\text { QUINTILE }\end{array}$} \\
\hline & $\begin{array}{l}\text { Männer } \\
\text { M5 }\end{array}$ & $\begin{array}{l}\text { Frauen } \\
\text { M5 }\end{array}$ & $\begin{array}{l}\text { Männer } \\
\text { M6 }\end{array}$ & $\begin{array}{l}\text { Frauen } \\
\text { M6 }\end{array}$ & $\begin{array}{l}\text { Männer } \\
\text { M7 }\end{array}$ & $\begin{array}{l}\text { Frauen } \\
\text { M7 }\end{array}$ \\
\hline \multicolumn{7}{|c|}{ Anteil Mehrarbeit im Beruf(Ref.: 1. Quintil) } \\
\hline \multirow[t]{2}{*}{ 2. Quintil } & - & - & - & - & $-0,432$ & $-0,148$ \\
\hline & - & - & - & - & $(0,330)$ & $(0,177)$ \\
\hline \multirow[t]{2}{*}{ 3. Quintil } & - & - & - & - & $-0,282$ & 0,064 \\
\hline & - & - & - & - & $(0,335)$ & $(0,190)$ \\
\hline \multirow[t]{2}{*}{ 4. Quintil } & - & - & - & - & $-0,962 *$ & $0,545^{*}$ \\
\hline & - & - & - & - & $(0,392)$ & $(0,252)$ \\
\hline \multirow[t]{2}{*}{ 5. Quintil } & - & - & - & - & $-0,786+$ & 0,052 \\
\hline & - & - & - & - & $(0,464)$ & $(0,252)$ \\
\hline \multicolumn{7}{|c|}{ Anteil ohne Heimarbeit im Beruf (Ref.: 1. Quintil) } \\
\hline \multirow[t]{2}{*}{ 2. Quintil } & - & - & - & - & $-0,283$ & 0,076 \\
\hline & - & - & - & - & $(0,345)$ & $(0,235)$ \\
\hline \multirow[t]{2}{*}{ 3. Quintil } & - & - & - & - & 0,017 & 0,120 \\
\hline & - & - & - & - & $(0,620)$ & $(0,476)$ \\
\hline \multirow[t]{2}{*}{ 4. Quintil } & - & - & - & - & $-0,645$ & $-0,674$ \\
\hline & - & - & - & - & $(0,817)$ & $(0,682)$ \\
\hline \multirow[t]{2}{*}{ 5. Quintil } & - & - & - & - & $-0,886$ & $-0,576$ \\
\hline & - & - & - & - & $(0,914)$ & $(0,724)$ \\
\hline \multicolumn{7}{|l|}{ Kontrollvariablen } \\
\hline \multicolumn{7}{|c|}{ Bildung (Ref.: mittel) } \\
\hline \multirow[t]{2}{*}{ Niedrig } & 0,291 & 0,004 & 0,303 & 0,011 & 0,292 & $-0,013$ \\
\hline & $(0,283)$ & $(0,214)$ & $(0,289)$ & $(0,214)$ & $(0,274)$ & $(0,215)$ \\
\hline \multirow[t]{2}{*}{ Hoch } & 0,136 & 0,121 & 0,111 & 0,138 & 0,111 & 0,097 \\
\hline & $(0,200)$ & $(0,131)$ & $(0,200)$ & $(0,130)$ & $(0,200)$ & $(0,129)$ \\
\hline \multirow[t]{2}{*}{ Berufserfahrung } & $-0,004 * *$ & $-0,003 * * *$ & $-0,004 * *$ & $-0,003^{* *}$ & $-0,004 * *$ & $-0,003 * * *$ \\
\hline & $(0,001)$ & $(0,001)$ & $(0,001)$ & $(0,001)$ & $(0,001)$ & $(0,001)$ \\
\hline \multirow{2}{*}{$\begin{array}{l}\text { Frühere } \\
\text { Teilzeittätigkeiten }\end{array}$} & 0,163 & $0,783^{* * *} *$ & 0,164 & $0,784 * * *$ & 0,171 & $0,789 * * *$ \\
\hline & $(0,192)$ & $(0,149)$ & $(0,191)$ & $(0,148)$ & $(0,191)$ & $(0,149)$ \\
\hline \multirow[t]{2}{*}{ Berufswechsel } & $1,401 * * *$ & $0,847 * * *$ & $1,376 * * *$ & $0,845^{* * *}$ & $1,387 * * *$ & $0,850 * * *$ \\
\hline & $(0,208)$ & $(0,127)$ & $(0,207)$ & $(0,127)$ & $(0,209)$ & $(0,125)$ \\
\hline \multirow[t]{2}{*}{ Leitungsposition } & $-0,768 * * *$ & $-0,191^{*}$ & $-0,779 * * *$ & $-0,204^{*}$ & $-0,823 * * *$ & $-0,190 *$ \\
\hline & $(0,225)$ & $(0,089)$ & $(0,225)$ & $(0,089)$ & $(0,228)$ & $(0,089)$ \\
\hline \multirow{2}{*}{$\begin{array}{l}\text { Arbeitsort: } \\
\text { Ostdeutschland }\end{array}$} & $-0,195$ & $-0,280^{* *}$ & $-0,193$ & $-0,280^{* *}$ & $-0,199$ & $-0,275 * *$ \\
\hline & $(0,176)$ & $(0,097)$ & $(0,175)$ & $(0,097)$ & $(0,176)$ & $(0,097)$ \\
\hline
\end{tabular}


Tab. 6 (Fortsetzung)

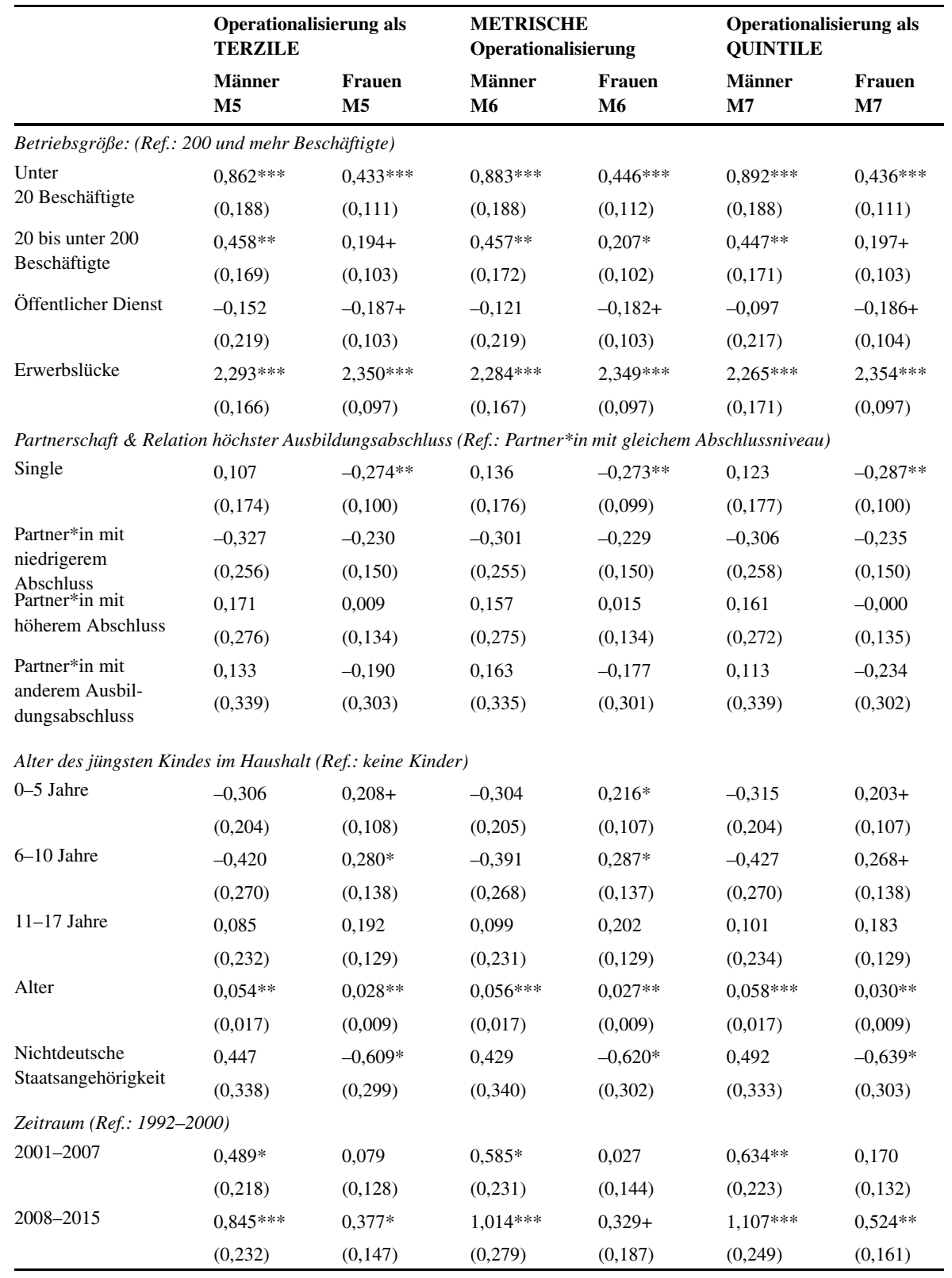


Tab. 6 (Fortsetzung)

\begin{tabular}{|c|c|c|c|c|c|c|}
\hline & \multicolumn{2}{|c|}{$\begin{array}{l}\text { Operationalisierung als } \\
\text { TERZILE }\end{array}$} & \multicolumn{2}{|c|}{$\begin{array}{l}\text { METRISCHE } \\
\text { Operationalisierung }\end{array}$} & \multicolumn{2}{|c|}{$\begin{array}{l}\text { Operationalisierung als } \\
\text { QUINTILE }\end{array}$} \\
\hline & $\begin{array}{l}\text { Männer } \\
\text { M5 }\end{array}$ & $\begin{array}{l}\text { Frauen } \\
\text { M5 }\end{array}$ & $\begin{array}{l}\text { Männer } \\
\text { M6 }\end{array}$ & $\begin{array}{l}\text { Frauen } \\
\text { M6 }\end{array}$ & $\begin{array}{l}\text { Männer } \\
\text { M7 }\end{array}$ & $\begin{array}{l}\text { Frauen } \\
\text { M7 }\end{array}$ \\
\hline \multicolumn{7}{|c|}{ Interaktionseffekte mit der Zeit } \\
\hline \multirow[t]{2}{*}{ Berufswechsel } & $0,011^{* *}$ & $0,007 * * *$ & $0,011 * *$ & $0,007 * * *$ & $0,011 * *$ & $0,008 * * *$ \\
\hline & $(0,003)$ & $(0,002)$ & $(0,003)$ & $(0,002)$ & $(0,003)$ & $(0,002)$ \\
\hline \multirow[t]{2}{*}{ Leitungsposition } & $0,009 * *$ & - & $0,009 * *$ & - & $0,010 * *$ & - \\
\hline & $(0,003)$ & - & $(0,003)$ & - & $(0,004)$ & - \\
\hline \multirow{2}{*}{$\begin{array}{l}\text { Frühere } \\
\text { Teilzeittätigkeiten }\end{array}$} & - & $-0,011^{* * *}$ & - & $-0,011^{* * *}$ & - & $-0,011^{* * *}$ \\
\hline & - & $(0,003)$ & - & $(0,003)$ & - & $(0,003)$ \\
\hline \multirow{2}{*}{$\begin{array}{l}\text { Anteil Mehrarbeit } \\
\text { im Beruf: 4. Quintil }\end{array}$} & - & - & - & - & - & $-0,005+$ \\
\hline & - & - & - & - & - & $(0,003)$ \\
\hline$N$ Monate & 672.892 & 415.852 & 672.892 & 415.852 & 672.892 & 415.852 \\
\hline$N$ Episoden & 12.061 & 7714 & 12.061 & 7714 & 12.061 & 7714 \\
\hline$N$ Übergänge & 263 & 717 & 263 & 717 & 263 & 717 \\
\hline$N$ Personen & 5147 & 3911 & 5147 & 3911 & 5147 & 3911 \\
\hline Log Likelihood & $-780,968$ & $-2452,827$ & $-785,135$ & $-2456,062$ & $-776,513$ & $-2444,831$ \\
\hline$A I C$ & 1625,936 & 4969,654 & 1628,270 & 4970,125 & 1631,026 & 4969,662 \\
\hline $\mathrm{Chi}^{2}$ & 626,745 & 1502,460 & 595,798 & 1482,764 & 664,283 & 1536,625 \\
\hline Pseudo $\mathrm{R}^{2}$ & 0,259 & 0,211 & 0,255 & 0,209 & 0,264 & 0,213 \\
\hline
\end{tabular}

Koeffizienten, robuste Standardfehler in Klammern ${ }^{+} p<0,1,{ }^{*} p<0,05,{ }^{* *} p<0,01,{ }^{* * *} p<0,001$

\section{Literatur}

Acker, Joan. 1990. Hierarchies, jobs, bodies. A theory of gendered organizations. Gender \& Society 4:139-158.

Allmendinger, Jutta, Corinna Kleinert, Reinhard Pollak, Basha Vicari, Oliver Wölfel, Agnieszka Althaber, Manfred Antoni, Bernhard Christoph, Katrin Drasch, Florian Janik, Ralf Künster, Marie-Christine Laible, Kathrin Leuze, Britta Matthes, Michael Ruland, Benjamin Schulz und Annette Trahms. 2019. Adult education and lifelong learning. In Education as a Lifelong Process, Hrsg. Hans-Peter Blossfeld und Hans-Günther Roßbach, 325-346. Wiesbaden: Springer.

Althaber, Agnieszka. 2018. Die Suche nach Gemeinsamkeiten. Strukturelle Gründe für die Teilzeitarbeit von Frauen und Männern. WZB-Mitteilungen 161:17-20.

Bächmann, Ann-Christin, und Dörthe Gatermann. 2017. The duration of family-related employment interruptions - the role of occupational characteristics. Journal for Labour Market Research 50:143-160.

Bechmann, Sebastian, Vera Dahms, Nikolai Tschersich, Marek Frei, Ute Leber und Barbara Schwengler. 2013. Beschäftigungsmuster von Frauen und Männern. Auswertungen des IAB-Betriebspanels 2012. IAB-Forschungsbericht 14/2013. Nürnberg.

Bell, Linda A., und Richard B. Freeman. 2001. The incentive for working hard: explaining hours worked differences in the US and Germany. Labour Economics 8:181-202.

Blossfeld, Hans-Peter, Katrin Golsch und Götz Rohwer. 2007. Techniques of event history modeling. New approaches to causal analysis. Mahwah, N.J: Erlbaum.

Blossfeld, Hans-Peter, Hans-Günther Roßbach und Jutta von Maurice, Hrsg. 2011. Education as a lifelong process. The German National Educational Panel Study (NEPS). Zeitschrift für Erziehungswissenschaft Sonderheft, Bd. 14. Wiesbaden: VS-Verlag für Sozialwissenschaften.

Boll, Christina. 2010. Lohneinbußen von Frauen durch geburtsbedingte Erwerbsunterbrechungen. Wirtschaftsdienst 90:700-702.

Brenke, Karl. 2011. Anhaltender Strukturwandel zur Teilzeitbeschäftigung. DIW Wochenbericht 42:3-12. 
Brines, Julie. 1994. Economic dependency, gender, and the division of labor at home. American Journal of Sociology 100:652-688.

Bühlmann, Felix, Guy Elcheroth und Manuel Tettamanti. 2010. The division of labour among European couples: The effects of life course and welfare policy on value-practice configurations. European Sociological Review 26:49-66.

Bünning, Mareike. 2016. Die Vereinbarkeitsfrage für Männer. Welche Auswirkungen haben Elternzeiten und Teilzeitarbeit auf die Stundenlöhne von Vätern? Kölner Zeitschrift für Soziologie und Sozialpsychologie 68:597-618.

Busch, Anne. 2013. Der Einfluss der beruflichen Geschlechtersegregation auf den „Gender Pay Gap“. KZfSS Kölner Zeitschrift für Soziologie und Sozialpsychologie 65:301-338.

Cassirer, Naomi. 2004. Change in Part-time Work across Occupations, 1970-1990. Research in Social Stratification and Mobility 20:145-184.

Cha, Youngjoo. 2013. Overwork and the persistence of gender segregation in occupations. Gender \& Society 27:158-184.

Cleves, Mario. 1999. Analysis of multiple failure-time data with Stata. Stata Technical Bulletin 9:30-39.

Dieckhoff, Martina, Vanessa Gash, Antje Mertens und Laura Romeu Gordo. 2016. A stalled revolution? What can we learn from women's drop-out to part-time jobs: A comparative analysis of Germany and the UK. Research in Social Stratification and Mobility 46:129-140.

Eichhorst, Werner, Paul Marx und Verena Tobsch. 2013. Non-standard employment across occupations in Germany: The role of replaceability and labour market flexibility. Discussion Paper 7662. Bonn.

Frommert, Dina, und Susanne Strauß. 2013. Biografische Einflussfaktoren auf den Gender Pension Gap Ein Kohortenvergleich für Westdeutschland. Journal for Labour Market Research 46:145-166.

Fuchs Epstein, Cynthia, Carroll Seron, Bonnie Oglensky und Robert Sauté. 1999. The Part-Time Paradox: Time norms, professional life, family and gender. London: Routledge.

Glass, Jennifer, und Tetsushi Fujimoto. 1995. Employer characteristics and the provision of family responsive policies. Work and Occupations 22:380-411.

Glauber, Rebecca. 2011. Limited access: Gender, occupational composition, and flexible work scheduling. The Sociological Quarterly 52:472-494.

Grunow, Daniela, Silke Aisenbrey und Marie Evertsson. 2011. Familienpolitik, Bildung und Berufskarrieren von Müttern in Deutschland, USA und Schweden. Kölner Zeitschrift für Soziologie 63:395-430.

Grunow, Daniela, Heather Hofmeister und Sandra Buchholz. 2006. Late 20th-century persistence and decline of the female homemaker in Germany and the United States. International Sociology 21:101-131.

Hägglund, Anna E., und Ann-Christin Bächmann. 2017. Fast lane or down the drain? Does the occupation held prior to unemployment shape the transition back to work? Research in Social Stratification and Mobility 49:32-46.

Hausmann, Ann-Christin, und Corinna Kleinert. 2014. Berufliche Segregation auf dem Arbeitsmarkt. Männer- und Frauendomänen kaum verändert. IAB-Kurzbericht 9/2014: Institut für Arbeitsmarkt- und Berufsforschung.

Hinz, Thomas, und Thomas Schübel. 2001. Geschlechtersegregation in deutschen Betrieben. Mitteilungen aus der Arbeitsmarkt- und Berufsforschung 34:286-301.

Hipp, Lena, und Kathrin Leuze. 2015. Institutionelle Determinanten einer partnerschaftlichen Aufteilung von Erwerbsarbeit in Europa und den USA. Kölner Zeitschrift für Soziologie und Sozialpsychologie 67:659-684.

Hipp, Lena, und Stefan Stuth. 2013. Management und Teilzeit? - Eine empirische Analyse zur Verbreitung von Teilzeitarbeit unter Managerinnen und Managern in Europa. Kölner Zeitschrift für Soziologie und Sozialpsychologie 65:101-128.

Institut Arbeit und Qualifikation. 2019. Sozialversicherungspflichtige Vollzeitbeschäftigung und Teilzeitbeschäftigung 2000-2018. http://www.sozialpolitik-aktuell.de/tl_files/sozialpolitik-aktuell/_ Politikfelder/Arbeitsmarkt/Datensammlung/PDF-Dateien/abbIV8c.pdf. Zugegriffen: 19. August 2019.

Kelly, Erin L., Samantha K. Ammons, Kelly Chermack und Phyllis Moen. 2010. Gendered challenge, gendered response. Confronting the ideal worker norm in a white-collar organization. Gender \& Society 24:281-303.

Klenner, Christina, und Yvonne Lott. 2016. Arbeitszeitoptionen im Lebensverlauf. Bedingungen und Barrieren ihrer Nutzung im Betrieb. Study 004. Düsseldorf: Wirtschafts- und Sozialwissenschaftliches Institut.

Klenner, Christina, und Tanja Schmidt. 2011. Teilzeitarbeit im Lebensverlauf von abhängig beschäftigten Frauen. In Neue Wege - Gleiche Chancen. Expertisen zum Ersten Gleichstellungsbericht der Bundes- 
regierung, Hrsg. Ute Klammer und Markus Motz, 253-311. Wiesbaden: VS Verlag für Sozialwissenschaften.

Konietzka, Dirk, und Michaela Kreyenfeld. 2010. The growing educational divide in mothers' employment: An investigation based on the German micro-censuses 1976-2004. Work, Employment and Society 24:260-278.

König, Pascal, Sebastian Jäckle und Georg Wenzelburger. 2014. Weiterführende statistische Methoden für Politikwissenschaftler: Eine anwendungsbezogene Einführung mit Stata. München: De Gruyter.

Krüger, Helga. 1995a. Dominanzen im Geschlechterverhältnis. Zur Institutionalisierung von Lebensläufen. In Das Geschlechterverhältnis als Gegenstand der Sozialwissenschaften, Hrsg. Regina BeckerSchmidt und Gudrun-Axeli Knapp, 195-219. Frankfurt a. M.: Campus.

Krüger, Helga. 1995b. Prozessuale Ungleichheit. Geschlecht und Institutionenverknüpfung im Lebenslauf. In Sozialstruktur und Lebenslauf. Sozialstrukturanalyse, Bd. 5, Hrsg. Peter A. Berger und Peter Sopp, 133-154. Wiesbaden: VS Verlag für Sozialwissenschaften.

Krüger, Helga. 2001. Ungleichheit und Lebenslauf. Wege aus den Sackgassen empirischer Traditionen. In Geschlechtersoziologie, Kölner Zeitschrift für Soziologie und Sozialpsychologie, Sonderheft 41, Hrsg. Bettina Heintz, 512-537. Opladen: Westdeutscher Verlag.

Kühhirt, M. 2012. Childbirth and the long-term division of labour within couples: How do substitution, bargaining power, and norms affect parents' time allocation in West Germany? European Sociological Review 28:565-582.

Leuze, Kathrin, und Susanne Strauß. 2016. Why do occupations dominated by women pay less? How 'female-typical' work tasks and working-time arrangements affect the gender wage gap among higher education graduates. Work, Employment and Society 30:802-820.

Matthes, Britta, Carola Burkert und Wolfgang Biersack. 2008. Berufssegmente: Eine empirisch fundierte Neuabgrenzung vergleichbarer beruflicher Einheiten. IAB-Discussion Paper 35/2008. Nürnberg: IAB.

Organisation for Economic Co-operation and Development. 2018. OECD Labour Force Statistics 2017. Paris: OECD Publishing.

Rengers, Martina. 2015. Unterbeschäftigung, Überbeschäftigung und Wunscharbeitszeiten in Deutschland. Ergebnisse für das Jahr 2014. WISTA 6. Wiesbaden.

Rudman, Laurie A., und Kris Mescher. 2013. Penalizing men who request a family leave: Is flexibility stigma a femininity stigma? Journal of Social Issues 69:322-340.

Schober, Pia S. 2013. The parenthood effect on gender inequality: Explaining the change in paid and domestic work when British couples become parents. European Sociological Review 29:74-85.

Solga, Heike, und Dirk Konietzka. 1999. Occupational matching and social stratification: Theoretical insights and empirical observations taken from a German-German comparison. European Sociological Review 15:25-47.

Steiber, Nadia, und Barbara Haas. 2012. Advances in explaining women's employment patterns. SocioEconomic Review 10:343-367.

Trappe, Heike, Matthias Pollmann-Schult und Christian Schmitt. 2015. The rise and decline of the male breadwinner model: institutional underpinnings and future expectations. European Sociological Review 31:230-242.

Vandello, Joseph A., Vanessa E. Hettinger, Jennifer K. Bosson und Jasmine Siddiqi. 2013. When equal isn't really equal: The masculine dilemma of seeking work flexibility. Journal of Social Issues 69:303-321.

Wanger, Susanne. 2011. Ungenutzte Potenziale in der Teilzeit. Viele Frauen würden gerne länger arbeiten. IAB-Kurzbericht 09/2011. Nürnberg: IAB.

Wanger, Susanne. 2015. Frauen und Männer am Arbeitsmarkt: Traditionelle Erwerbs- und Arbeitszeitmuster sind nach wie vor verbreitet. IAB-Kurzbericht 4/2015. Nürnberg.

Williams, Christine L., Chandra Muller und Kristine Kilanski. 2012. Gendered Organizations in the New Economy. Gender \& Society 26:549-573.

Williams, Joan C., Mary Blair-Loy und Jennifer L. Berdahl. 2013. Cultural schemas, social class, and the flexibility stigma. Journal of Social Issues 69:209-234.

Wolf, Elke. 2014. The German part-time wage gap: Bad news for men? SOEPpapers on Multidisciplinary Panel Data Research No. 663.

Agnieszka Althaber 1984, Diplom-Soziologin, wissenschaftliche Mitarbeiterin am Wissenschaftszentrum Berlin für Sozialforschung (WZB) und Doktorandin an der Freien Universität Berlin. Forschungsgebiete: Lebensverlaufsforschung, Geschlechterungleichheiten, Arbeitsmarkt- und Berufsforschung. 
Kathrin Leuze 1975, Dr. rer. pol., Professorin für Methoden der empirischen Sozialforschung und Sozialstrukturanalyse, Friedrich-Schiller-Universität Jena. Forschungsgebiete: Lebenslaufforschung mit den Schwerpunkten Hochschulbildung, Arbeitsmarkt- und Berufsforschung, Geschlechterungleichheit. Veröffentlichungen: Why do occupations dominated by women pay less? How 'female-typical' work tasks and working time arrangements affect the gender wage gap among higher education graduates. Work, Employment and Society 30, 2016 (mit S. Strauß); Entwertung von Frauenberufen oder Entwertung von Frauen im Beruf? Eine Längsschnittanalyse zum Zusammenhang von beruflicher Geschlechtersegregation und Lohnentwicklung in Westdeutschland. Kölner Zeitschrift für Soziologie und Sozialpsychologie 67, 2015 (mit A.-C. Hausmann und C. Kleinert). 